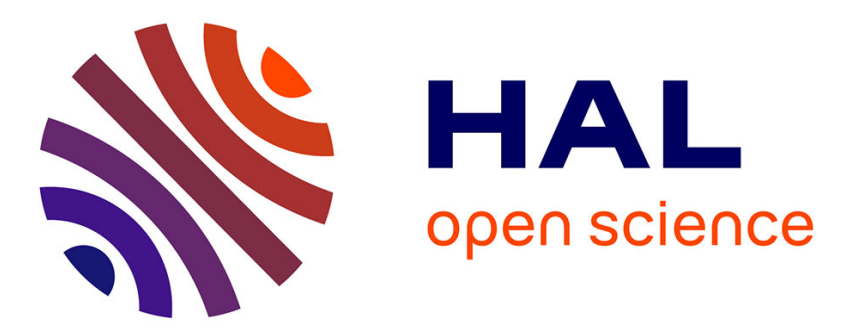

\title{
Variability and singularity arising from poor compliance in a pharmacokinetic model I: the multi-IV case
}

Pierre-Emmanuel Lévy Véhel, Jacques Lévy Véhel

\section{To cite this version:}

Pierre-Emmanuel Lévy Véhel, Jacques Lévy Véhel. Variability and singularity arising from poor compliance in a pharmacokinetic model I: the multi-IV case. Journal of Pharmacokinetics and Pharmacodynamics, 2013, 40 (1), pp.15-39. hal-00752114

\section{HAL Id: hal-00752114 \\ https://hal.inria.fr/hal-00752114}

Submitted on 14 Nov 2012

HAL is a multi-disciplinary open access archive for the deposit and dissemination of scientific research documents, whether they are published or not. The documents may come from teaching and research institutions in France or abroad, or from public or private research centers.
L'archive ouverte pluridisciplinaire HAL, est destinée au dépôt et à la diffusion de documents scientifiques de niveau recherche, publiés ou non, émanant des établissements d'enseignement et de recherche français ou étrangers, des laboratoires publics ou privés. 


\title{
Variability and singularity arising from poor compliance in a pharmacokinetic model I: the multi-IV case
}

\author{
P.E. Lévy Véhel \\ Laboratoire J.-A. Dieudonné, Université de Nice, France \\ pierem@math.unice.fr \\ and \\ J. Lévy Véhel \\ Regularity team, INRIA Saclay-Ile-de-France, Orsay, France \\ jacques.levy-vehel@inria.fr
}

\begin{abstract}
We consider a simple multi-IV model for drug concentration in the case of poor patient compliance. The model is a stochastic one, and is thus able to take into account an irregular drug intake schedule. Under some assumptions, we study features of the drug concentration relevant for practical purposes such as its variability or the regularity of its cumulative probability distribution. We consider five variants: random instants for drug intake with either deterministic or random doses, both in continuous and discrete-time settings, plus a model with stochastically varying elimination rate. Our computations make it possible to assess in a precise way the effect of various significant parameters such as the mean rate of intake, the elimination rate, and the mean dose. They also quantify how much poor compliance will affect the regimen: in that view, we provide precise comparisons with the variability of concentration in the cases of (a) a fully compliant patient and (b) a population of fully compliant patients with lognormally distributed elimination rates. The time discretized version of our models reveal unexpected links with measures known as infinite Bernoulli convolutions. Our findings help in understanding the consequences of poor compliance, and may have practical outcomes in terms of drug dosing and scheduling.
\end{abstract}




\section{Introduction}

Poor adherence to medical treatment is a problem of the utmost importance that has critical impact on the efficacy of therapy, particularly in the case of chronic diseases [20]. Compliance to pharmacotherapy can range from $5 \%$ to $90 \%$ [7]. Because of this, therapies tested on strictly adhering patients may reveal ineffective in ambulatory settings: as shown in [9], the drug concentration time evolution is heavily influenced by the random drug input generated by patients with poor adherence behaviour.

In the seminal work [9], the authors attacked the problem of mathematically modelling poor compliance using a probabilistic frame. They considered general distributions for the random instants of drug intake and studied the mean and variance of the concentration conditioned on the time elapsed since the last intake. Our work is similar in spirit. The present article is the first in a series of three where we will consider models of increasing generality and complexity. More precisely, we investigate below the probability distribution of drug concentration in the context of multiple-IV dosing and poor compliance: patients takes their drugs at random times, with possibly random doses. In this work, we use the simplest possible law to model the random times of drug intake, i.e. a homogeneous Poisson distribution. In other words, the moments of drug intake are supposed to follow a Poisson process. This assumption allows one to perform explicit computations using the well-developed machinery on Poisson processes, and to obtain precise results describing various aspects of the distribution of the concentration that are important for assessing the efficacy of the regimen. Indeed, we focus on two aspects of practical relevance: the variability of the concentration and the regularity of its probability distribution. It is intuitively obvious that poor compliance will increase the variability of the concentration around its mean as compared to the full compliance case. In addition, it may also have an impact on the regularity of the distribution of the concentration, resulting in a high probability of having too small a concentration of drugs. Our results quantify this in a precise way, showing the exact role played by each parameter of the process.

An even more radical situation occurs if, instead of considering a continuous time model, one investigates a time-discretized version, where doses may be taken only at discrete times: in this setting, the problem at hand reveals unexpected links with mathematical objects that have been studied in the literature for over seventy years under the name of infinite Bernoulli convolutions. Again, depending on some parameters, the discretized concentration may exhibit an extremely irregular 
behaviour. In effect, this means that the probability of observing a concentration $C$ depends in a very non-smooth way on the precise value of $C$. This is obviously an undesirable feature which may have strongly negative consequences.

We compare the variability induced by non compliance to the one induced by a distribution of elimination rates in a population. This helps putting in perspective the negative impacts of poor compliance.

We also consider the case where not only the intake times and doses are random, but also is the elimination rate across time. While this is not strictly related to poor compliance, such an analysis allows one to draw interesting conclusions, by comparing the effects of poor compliance to the ones due to a time varying elimination rate.

We do not address in this work the problem of estimating the parameters of the various models, in particular the law of the random intake times and doses. A recent article dealing with this issue is [2].

We end this introduction with a word on our modelling choices: clearly, the use of the multiple-IV model as well as the one of the homogeneous Poisson law for the random instants of intake are somewhat restrictive. Indeed, the problem of irregular drug-intake intervals is more likely to happen in the frame of oral administration than in the IV case. Likewise, the irregular schedule ought to be too complex in general to be described satisfactorily by a homogeneous Poisson law. However, we believe that our choice of describing results in the multiple-IV model / Poisson law as a first step are justified by the following facts:

- The essential theoretical ideas are already present in this simplified setting, allowing one to grasp the main mechanisms through which poor compliance impacts concentration.

- The mathematical apparatus, although still reasonably simple, draws on a few advanced concepts and requires sometimes lengthy computations. They serve as a intermediate step for the more complex analysis needed in the oral case / general schedule.

- The results presented below already help understanding the practical consequences of poor compliance, and may be used as rough guidelines for drug dosing and scheduling.

We will go beyond these limitations in two sequels of this work : in the first one, we will study the more realistic multi-oral model, and deal with the complications brought by the first-order kinetics, which are essentially technical. The 
second one will consider a more powerful mathematical frame, known as Piecewise Deterministic Markov Model, that will allow us to deal with general drug intake schedules, going beyond the homogeneous Poisson case.

The remaining of this work is organized as follows: for the reader's convenience, we present an overview of our main findings pertaining to the various models and their practical implications in the next section. A reader not interested in the mathematical details may concentrate on this section to get a quick summary of our work, and refer if needed to the details in the following sections. In Section 3, we set up the basic continuous time model for deterministic dose random time drug intake and study its variability (Subsection 3.1) and regularity (Subsection 3.2). In Section 4, we analyse the random doses version of this model. As before, we study its variability (Subsection 4.1) and regularity (Subsection 4.2). We present the discrete-time version of this model in section 5. We derive the discrete time concentration in Subsection 5.1 and study its variability in Subsection 5.2. In Subsection 5.3, we show that the discrete time model tends to the continuous time one when the discretization step tends to 0. Subsection 5.4 describes the complex regularity behaviour of the cumulative probability distribution of the concentration in the discrete time model. Section 6 parallels this for the discrete time random dose case. Finally, Section 7 follows the same pattern in the case of discrete time, random dose, and random elimination rate. The appendix in Section 8 contains the proofs of some of the more technical mathematical results.

\section{Overview: pharmokinetic implications of non com- pliance}

For convenience, we present in this section an overview of the random models we consider to account for non-compliance, as well as their practical pharmokinetic consequences. We concentrate on two aspects that are of direct relevance for assessing the impact of non-compliance: variability and regularity of concentration. Variability characterizes the probability that the random concentration becomes "large" or "small", as compared to its expected values. We typically measure variability by the variance of the random concentration, but consider other features as well. Regularity indicates whether the concentration varies smoothly or not. More precisely, the concentrations encountered in our models may be either: (a) regular, which heuristically means that the probabilities of observing concentrations $C_{1}$ and $C_{2}$ are similar if $C_{1}$ and $C_{2}$ are close ; (b) singular at 0 , indicating 
that observing a vanishing concentration has a very large probability ; (c) everywhere singular, which means that certain values of concentration have a high probability to occur, while others, which may be almost the same, will happen rarely. In other words, concentration in this case may vary wildly between patients. This is obviously an undesirable feature, as, for instance, it makes it more difficult to characterize a mean behaviour. Another practical negative implication of a singular distribution is that such a distribution will be difficult to approximate numerically. Indeed, it is a well-known fact in non-parametric statistics (see e.g. [19]) that the convergence rate of usual estimators for probability distributions (such as histograms or kernel estimators) worsens when the target distribution is singular.

In summary, variability of the concentration characterizes how far the concentration typically departs from its expected one, while regularity of the concentration refers to the way it varies, i.e. smoothly or in an abrupt way.

We study these two features in five variants of non compliance: (1) a continuous time model where only the time of intakes are random, (2) the same model but where the doses are also allowed to be random, (3) a discrete time model (meaning that drug intake may only occur at some specific, regularly spaced, time instants) with random time intakes, (4) the same model where the doses are also allowed to be random, (5) model (4) where we also allow the elimination rate to vary randomly in time. Heuristically, the continuous time models correspond to situations where the treatment must be taken "in the morning", or "in the evening", while the discrete cases correspond to situations where the treatment must be taken at fixed times. These models are explained in full details respectively in Sections 3, 4, 5, 6 and 7. Moreover, we compare the variabilities in the models above with the ones in the cases of (a) full compliance of a single patient and (b) full compliance in a population where we take into account variability due to differing elimination rates or clearances between individuals. This allows us to highlight "equivalent scenarios" where, for instance, we find the parameters of a non-compliant patient that will yield the same variance in concentration as in a whole population with given distribution of elimination rates, or, which is the same, of a single individual whose elimination rate is unknown and is modelled as a random variable.

Before we present the non compliant models in Subsections 2.3, 2.4, 2.5, 2.6 and 2.7, we briefly analyse the variability in the fully compliant models for a single patient (Subsection 2.1) and a population with lognormally distributed elimination rates or clearances (Subsection 2.2). 


\subsection{Purely deterministic model (full compliance)}

As explained in the introduction, for simplicity and because it permits to convey the essential features of random non-compliance, we will only consider in this work (as is done in the first part of [9]) the case of random multidose intravenous (IV) administration. We deal with the simple one-compartment pharmacokinetic model with first-order elimination process of coefficient $k$ (the elimination rate), and apparent volume of distribution $V_{d}$. An analysis in the frame of a multiple oral model will be presented elsewhere.

We thus assume that a patient takes a fixed dose $D$ at regularly spaced times $t_{0}, t_{1}, \ldots, t_{N}, \ldots$, with $t_{i}=\frac{i}{\lambda}$, that is, the patient takes drugs every $\frac{1}{\lambda}$ units of time for some positive $\lambda$. These doses translate into immediate (i.e. at each time $t_{i}$ ) increases of the concentration by the value $\frac{D}{V_{d}}$. After that, the effect of the dose taken at $T_{i}$ on the overall concentration decreases exponentially fast, with exponential speed $k$. Formally, this means that, at time $t$, the drug concentration reads:

$$
C_{d}(t)=\frac{D}{V_{d}} \sum_{i=0}^{N(t)} \exp \left(-k\left(t-t_{i}\right)\right),
$$

where $t_{0}, t_{1}, \ldots, t_{N(t)}$ denote the instants of drug intake up to time $t$. The concentration may also be expressed as follows:

$$
C_{d}(t)=\frac{D}{V_{d}} \sum_{i=0}^{\infty} \exp \left(-k\left(t-t_{i}\right)\right) \mathbb{1}\left(t \geqslant t_{i}\right) .
$$

We will wish to compare the values of the variances in various stochastic models below to the one in the case of full compliance. Of course, in the frame of full compliance, there is no randomness involved, and one cannot define proper mean and variance. However, since the concentration varies in time, it makes sense to average it over all values of $t$, and define the variance correspondingly. In other words, we denote:

$$
\begin{gathered}
E_{d}=\lim _{T \rightarrow \infty} \frac{1}{T} \int_{0}^{T} C_{d}(t) d t \\
\operatorname{Var}_{d}=\lim _{T \rightarrow \infty} \frac{1}{T} \int_{0}^{T}\left(C_{d}(t)-E_{d}\right)^{2} d t
\end{gathered}
$$


for the mean and variance with respect to time of $C_{d}(t)$. Note that $E_{d}$ is closely related to the commonly measured pharmacokinetic metric AUC. As for $\operatorname{Var}_{d}$, it represents the time-averaged squared deviation from the long-term average, and quantifies the variability within a cycle in the steady state. In that sense it is analogous to (but different from) the "Peak trough fluctuation" parameter considered in the PK literature. Simple computations lead to:

$$
\begin{gathered}
E_{d}=\mu \frac{D}{V_{d}} \\
V a r_{d}=\frac{\mu}{2}\left(\frac{D}{V_{d}}\right)^{2} \frac{e^{\frac{1}{\mu}}-2 \mu e^{\frac{1}{\mu}}+2 \mu+1}{e^{\frac{1}{\mu}}-1},
\end{gathered}
$$

where $\mu=\frac{\lambda}{k}$. These quantities are related to ones considered in [10].

For comparison with the random models below, we note the following facts:

- For a fixed mean equal to 1 , the variance of $C_{d}$ tends to infinity at speed $\frac{1}{12 \mu^{2}}$ when $\mu \rightarrow 0$. The variance tends to 0 at speed $\frac{1}{2 \mu}$ when $\mu \rightarrow \infty$.

- $\mathbb{P}\left(\left|C_{d}-\mathbb{E}\left(C_{d}\right)\right| \geqslant \gamma \mathbb{E}\left(C_{d}\right)\right) \leqslant \frac{1}{12 \mu^{2} \gamma^{2}}$ for large $\mu$ (see Formula (17) in Subsection 3.1 for a more precise expression and Figure 3 for a comparison with a non compliant case).

These formulas quantify the obvious fact that, everything else being fixed, the variability of the concentration is a decreasing function of the number of takes per unit time. As we shall see, non compliance amplifies the the variability of the concentration, and we will use formula (3) to measure the strength of this amplification.

\subsection{Full compliance with population elimination rate variabil- ity}

So far we have considered the case of a single compliant subject, whose pharmacokinetic parameters are perfectly known. We now investigate the case of a whole population, among which the elimination rate $k$ presents some amount of variability. We assume moreover that each subject in the population is fully compliant, and that their drug intake frequency $\lambda$ is the same. This amounts to replacing $k$ by $k_{0} U_{k}$ in the preceding computations, where $U_{k}$ is a dimensionless random variate that represents the variability among subjects. We assume that $U_{k}$ follows a 
lognormal distribution with parameters ${ }^{1} m_{k}=0, \sigma_{k}^{2}$. The lognormal distribution is a common modelling assumption for positive continuous quantities, and seems to fit well with the findings of large-scale Pop-PK studies $([6,2])$. The choice $m_{k}=0$ ensures that the reference value $k_{0}$ is the median of the distribution.

We wish to examine the impact of this variability on the long-term average concentration $E_{d}$; recall that $E_{d}$ is closely related to the PK metric AUC. Let us first assume that other pharmacokinetic parameters such as $V_{d}$ are constant across the population, so that the variations in $E_{d}$ (hence also area under curve AUC and clearance $\mathrm{Cl}$ ) are only due to the variations of $k$. In other words, we consider the random variable $E_{d}=E_{d}\left(k_{0} U_{k}\right)$, where $U_{k}$ follows a lognormal distribution, and measure its variability.

An application of the classical formula for the moment-generating function for the Gaussian distribution (see [11]) shows that the mean and variance of $E_{d}$ across the population are given by :

$$
\begin{gathered}
\mathbb{E}_{\text {pop }}\left(E_{d}\right)=\frac{\lambda}{k_{0}} \frac{D}{V_{d}} \mathbb{E}_{\text {pop }}\left(\frac{1}{U_{k}}\right)=\frac{\lambda}{k_{0}} \frac{D}{V_{d}} e^{\sigma_{k}^{2} / 2}, \\
\operatorname{Var}_{\text {pop }}\left(E_{d}\right)=\frac{\lambda^{2}}{k_{0}^{2}} \frac{D^{2}}{V_{d}^{2}} \operatorname{Var}_{\text {pop }}\left(\frac{1}{U_{k}}\right)=\frac{\lambda^{2}}{k_{0}^{2}} \frac{D^{2}}{V_{d}^{2}} e^{\sigma_{k}^{2}}\left(e^{\sigma_{k}^{2}}-1\right) .
\end{gathered}
$$

These results can be rewritten in terms of the reduced parameter $\mu_{0}=\lambda / k_{0}$ and the coefficient of variation $\mathrm{CV}_{k}=\sqrt{e^{\sigma_{k}^{2}}-1}$ of the elimination rate:

$$
\begin{gathered}
\mathbb{E}_{\text {pop }}\left(E_{d}\right)=\mu_{0} \frac{D}{V_{d}} \sqrt{1+\mathrm{CV}_{k}^{2}}, \\
\operatorname{Var}_{\text {pop }}\left(E_{d}\right)=\mu_{0}^{2} \frac{D^{2}}{V_{d}^{2}} \mathrm{CV}_{k}^{2}\left(1+\mathrm{CV}_{k}^{2}\right) .
\end{gathered}
$$

The same approach applies if one wishes to take into account in addition variability of $V_{d}$ across the population: starting from the expression $E_{d}=\frac{\lambda}{k} \frac{D}{V_{d}}$, one simultaneously replaces $k$ by $k_{0} U_{k}$ and $V_{d}$ by $V_{d 0} U_{V}$, where $\left(U_{k}, U_{V}\right)$ is a dimensionless random vector. Inferring the distribution of $E_{d}$ from this expression requires the knowledge of the joint distribution of $U_{k}$ and $U_{V}$, or at least that of $U_{k} U_{V}$. Joint distributions are not usually reported in Pop-PK studies, but since the product $k V_{d}$ is nothing but the clearance $\mathrm{Cl}$, we can see that in that case:

$$
\mathrm{Cl}=k_{0} U_{k} V_{d 0} U_{V}=\mathrm{Cl}_{0} U_{\mathrm{Cl}}
$$

\footnotetext{
${ }^{1}$ We follow the classical convention in which the parameters $m, \sigma^{2}$ for the lognormal distribution are respectively the mean and variance of the logarithm of the random variable.
} 


$$
\begin{array}{|l|l|l|}
\hline D=10 m g & V_{d}=83 l & k=0.022 h^{-1} \\
\hline
\end{array}
$$

Table 1: Numerical values of the parameters used in numerical experiments.

where $\mathrm{Cl}_{0}=k_{0} V_{d 0}$ and $U_{\mathrm{Cl}}=U_{k} U_{V}$. The random variate $U_{\mathrm{Cl}}$ represents the variability of clearance, and its distribution is more frequently reported in Pop-PK studies. If we assume again a lognormal distribution with parameters $m_{\mathrm{Cl}}=0$ and $\sigma_{\mathrm{Cl}}^{2}$ around the median clearance $\mathrm{Cl}_{0}$ (consistent for instance with the results of [6]), the same computations yield:

$$
\begin{gathered}
\mathbb{E}_{p o p}\left(E_{d}\right)=\mu_{0} \frac{D}{V_{d}} e^{\sigma_{\mathrm{Cl}}^{2} / 2}, \\
\operatorname{Var}_{p o p}\left(E_{d}\right)=\mu_{0}^{2} \frac{D^{2}}{V_{d}^{2}} e^{\sigma_{\mathrm{Cl}}^{2}}\left(e^{\sigma_{\mathrm{Cl}}^{2}}-1\right) .
\end{gathered}
$$

We note in passing that lognormality of clearance reported in the literature is compatible with joint lognormality of $\left(k, V_{d}\right)$.

For consistency, we will always use in the sequel the parameters of [6] in comparing numerically the various models. These are recalled in Table 1, and yield an AUC equal to $5.48 \mathrm{mg} \times \mathrm{h} / \mathrm{l}$. Note that a mean elimination rate of $0.022 h^{-1}$ corresponds to a median $k_{0}=0.018 h^{-1}$. As an example, with a coefficient of variation $\mathrm{CV}_{k}=73 \%$ (as in [6]), which corresponds to a variance of 0.43 , and assuming that doses are taken every $\frac{1}{\lambda}=12 h$, the values of the mean and variance of $E_{d}$ are

$$
\mathbb{E}_{\text {pop }}\left(E_{d}\right)=0.69 \mathrm{mg} \cdot \mathrm{l}^{-1} \quad ; \quad \operatorname{Var}_{\text {pop }}\left(E_{d}\right)=0,25 \mathrm{mg} \cdot \mathrm{l}^{-2},
$$

where we have assumed for simplicity that $V_{d}$ is constant. We have plotted on Figure 1 eight sample evolutions of the concentration for the first 14 days using this scenario. This figure shows that the steady state (periodic concentration) is quickly reached, though the convergence can be a little slower for smaller realisations of $k$ (topmost curve).

In the following sections, we go back to the case of a single subject with perfectly known PK parameters but poorly compliant behaviour, and will compare the variability in the various cases. 


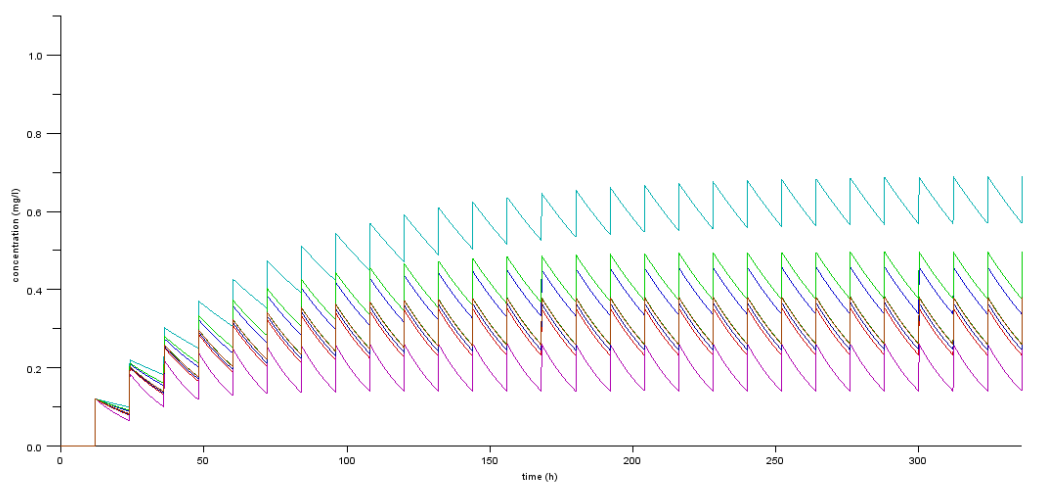

Figure 1: Eight sample paths of the concentration in the presence of elimination rate variability for the first 14 days.

\subsection{Continuous model with random intake instants and deter- ministic doses}

In the frame of poor compliance, the time instants of drug intake are modelled as random variables instead of being fixed. We shall denote these stochastic time instants as $\left(T_{i}\right)_{i \in \mathbf{N}}$ and assume that the random durations $T_{i}-T_{i-1}$ between two intakes are independent and identically distributed (hereafter abbreviated "i.i.d.") with exponential law of parameter $\lambda$. In other words, the sequence $\left(T_{i}\right)_{i \in \mathbf{N}}$ form a homogeneous Poisson process, and the mean duration between two drug intakes is equal to $\frac{1}{\lambda}$. The (stochastic) concentration at time $t$ reads:

$$
C(t)=\frac{D}{V_{d}} \sum_{i=0}^{\infty} \exp \left(-k\left(t-T_{i}\right)\right) \mathbb{1}\left(t \geqslant T_{i}\right) .
$$

This model is illustrated on Figure 2, which displays eight sample paths of the evolution of the concentration $C(t)$, simulated using the parameters given in Table 1 and $\lambda^{-1}=12 h$.

We show in Section 3 that $C(t)$ has a well defined limit when $t$ tends to infinity. We are interested in the variability and regularity properties of this steady state, or asymptotic concentration, that is denoted $C$. The following formulas are established in Section 3:

$$
\mathbb{E}(C)=\mu \frac{D}{V_{d}}
$$




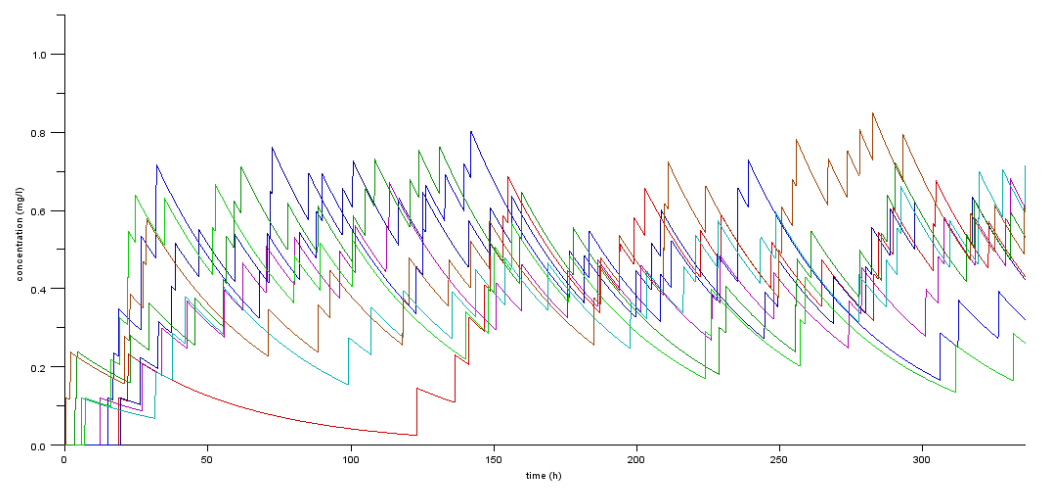

Figure 2: Eight sample paths of the concentration with Poisson distributed instants of intakes, for the first 14 days.

$$
\operatorname{Var}(C)=\frac{\mu}{2}\left(\frac{D}{V_{d}}\right)^{2} .
$$

In view of (2) and (3), we remark that:

- as expected, the means are the same in the deterministic and random models;

- for a fixed mean equal to 1 , the variance of $C$ is equal to $\frac{1}{2 \mu}$. It thus tends at this speed to 0 when $\mu \rightarrow 0$ (six times more slowly than in the deterministic model) and to infinity when $\mu \rightarrow \infty$ (same speed as in the deterministic model);

- $\mathbb{P}(|C-\mathbb{E}(C)| \geqslant \gamma \mathbb{E}(C)) \leqslant \frac{1}{2 \mu \gamma^{2}}$ for all $\mu$. We plot on Figure 3 a comparison between this bound and the one in the fully compliant case given by Formula (17) (see Subsection 3.1).

- the probability that the long-term concentration exceeds a given level $U$ decays as $\frac{1}{U^{2}}$. More precisely, $\mathbb{P}(C \geqslant U) \leqslant \frac{7}{6 U^{2}}\left(\frac{D}{V_{d}}\right)^{2} \mu\left(\mu+\frac{1}{2}\right)$. With $\mu \frac{D}{V_{d}}=\mathbb{E}(C)$ constant, one sees that this probability decays faster for larger values of $\mu$. 

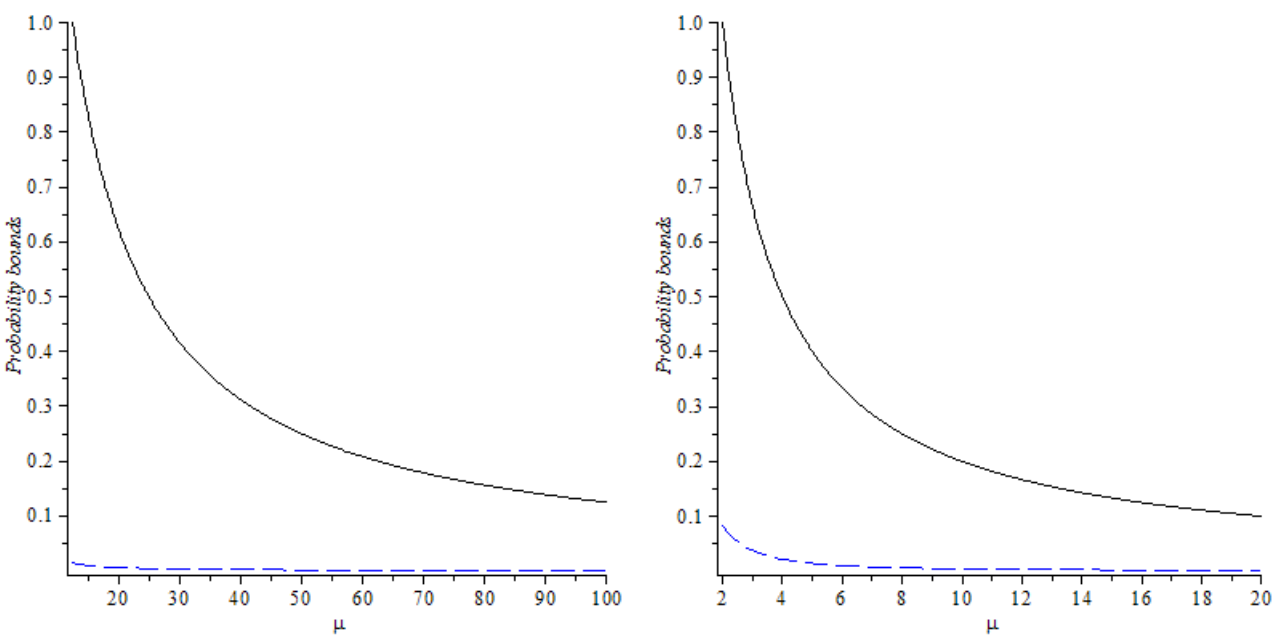

Figure 3: Probabilities that the concentration will significantly depart from its mean (Formulas (16) and (17)) in the cases of full compliance (dashed) and non compliance with random instants of intake (solid), as a function of $\mu$. Left: $\gamma=$ 0.2 , right: $\gamma=0.5$.

We see that, in a similar way as was observed in the deterministic model, the variability of the concentration is a decreasing function of the expected number of takes per unit time: as is intuitively clear, increasing the mean frequency of intakes while keeping constant the average quantity of administrated drug diminishes the negative impact of poor compliance in terms of the probability of departing significantly from the mean concentration. However, the same value of $\mu$ yields larger variability in the non-compliant case than in the deterministic one, in a way quantified by the formulas above. To illustrate the differences between the variances in the deterministic -formula (3)- and random cases -formula (10)-, we plot on Figures 4 and 5 their evolutions as a function of $\mu$, in two situations. First, we let $\mu$ vary and keep the other parameters, i.e. $D$ and $V_{d}$ constant. This corresponds for instance to the case where the number of doses per unit time (or average number of doses per unit time in the stochastic case) evolves, while maintaining everything else unchanged. One can see on Figure 4 that, when $\mu$ tends to infinity, the variance in the deterministic frame reaches a plateau (the limit value is $\frac{1}{12}\left(\frac{D}{V_{d}}\right)^{2}$ ), while the random situation leads to an unbounded variance. Hence non-compliance has here a dramatic effect. When $\mu$ tends to 0 , both variances 
behave as $\frac{\mu}{2}$ and thus tend to 0 . This simulation is however somewhat unrealistic since it leads to an unbounded increase of the mean concentration when $\mu$ tends to infinity. This is why, on Figure 5, we consider the case where $\mu$ varies but the mean is kept constant. From Formula (12) and (2), we see that this simply translates into ensuring $\mu \frac{D}{V_{d}}=$ constant. Here, we take $\mu \frac{D}{V_{d}}=0.23$, which corresponds to the parameters given in Table 1 , and $\lambda=1$ day. Figure 5 shows that both variances tend to 0 when $\mu$ tends to infinity. The speed of convergence is however faster in the deterministic case $\left(\frac{1}{12 \mu^{2}}\right)$ than in the random one $\left(\frac{1}{2 \mu}\right)$. When $\mu$ tend to 0 , both variances tend to infinity at same rate $\left(\frac{1}{2 \mu}\right)$.

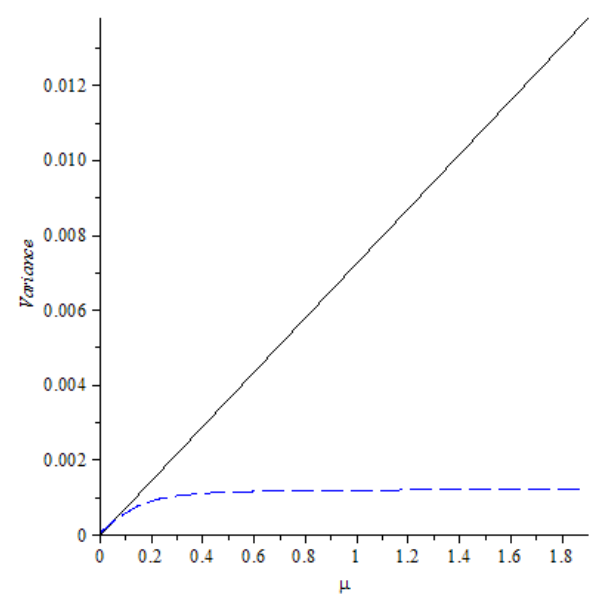

Figure 4: Evolution of the variance as a function of $\mu$ in the deterministic (dashed) and random (solid) cases, when all other parameters are kept constant. The value $\mu=1.9$ corresponds to $\lambda=1$ day.

A perhaps even more revealing comparison is to plot the values of $\lambda$ in the random model as a function of the ones of $\lambda$ in the deterministic one that yield the same variance: for instance, the point $(1 / 24,0.47)$ in the graph on Figure 6 means that a compliant patient taking a dose every day will have same concentration variability as a non compliant one taking in average a dose every $2.1=1 / 0.47$ hours, when the mean dose per day and all other parameters are the same in both situations.

Let us now present a comparison of the variance induced by non compliance in the random instants model to the one entailed by differing elimination rates in a fully compliant population. Similarly to Figure 6, we plot on Figure 7 the values 


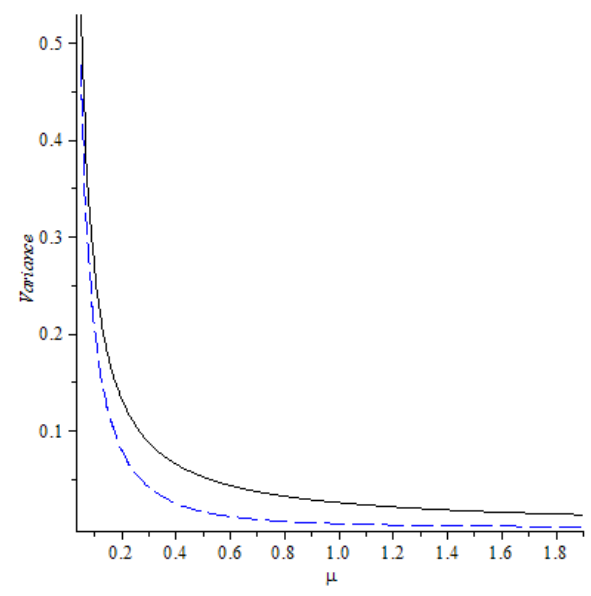

Figure 5: Evolution of the variance as a function of $\mu$ in the deterministic (dashed) and random (solid) cases, when the mean is kept constant.

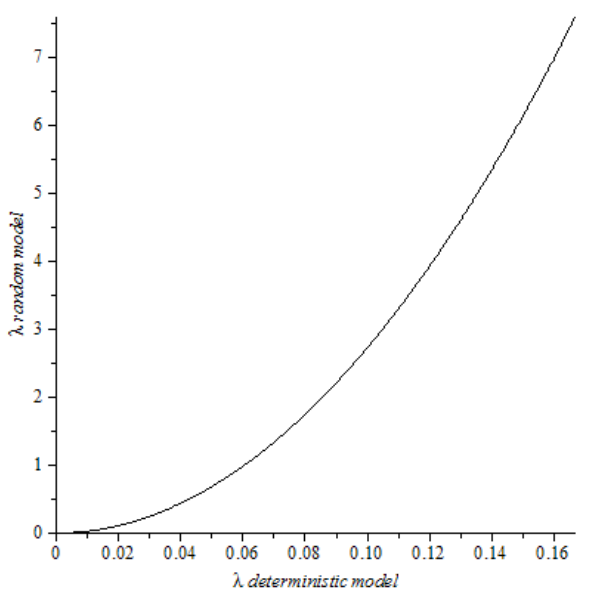

Figure 6: Average number of doses per hour in the non-compliant case as a function of the number of doses per hour in the fully compliant one yielding same variance for the concentration (elimination rate $k=0.022 h^{-1}$ ). 
of $\lambda$ in the random model as a function of the variance $\sigma_{k}^{2}$ of the elimination rates in the model of Section 2.2 that yield the same variance of concentration when the mean dose per day and all other parameters are the same in both situations. From Formulas (4), (5), (9) and (10), we see that this amounts to setting:

$$
\lambda=\frac{k}{2\left(e^{\sigma_{k}^{2}}-1\right)},
$$

where $\lambda$ is the mean number of doses per hour of the non compliant patient, $k$ is both his elimination rate and the mean elimination rate in the population, and $\sigma_{k}^{2}$ is the variance of the elimination rates in the population. As an example, the point $(0.43,0.02)$ on the graph means that the variance of concentration in a population of compliant patients were the variance in elimination rates in 0.43 (as in [6]) will be the same as for a single non compliant patient taking in average a dose every $49(=1 / 0.02)$ hours. Likewise, a single non compliant patient taking in average a dose every day displays the same variance in concentration as a population of fully compliant individuals with elimination rates variance equal to 0.24 , i.e. a coefficient of variation equal to $52 \%$.

Note that exactly the same conclusions (including the numerical values) hold if one considers a population where the clearance instead of only the elimination rate follows a lognormal distribution, as Formulas (4) and (5) on the one hand, and Formulas (6) and (7) on the other hand are similar upon replacing $\sigma_{k}$ with $\sigma_{\mathrm{Cl}}$.

Let us now move to the regularity properties of the distribution of $C$. We show in Section 3.2 that $\mu$ governs the smoothness of the distribution of the concentration: a larger $\mu$ translates into a better behaved concentration. More precisely, in the long term, the cumulative probability distribution of drug concentration may display two kinds of irregular behaviours. We just mention here that, when $\mu$ is larger than one, the distribution is regular, while, when it is smaller than one, it is singular at the origin. In practical terms, this amounts to quantifying in a precise way the situations where the moments of intakes are too scarce with respect to the elimination rate, resulting in a high probability of having too small a concentration of drugs.

To illustrate this fact, we plot on Figure 8 histograms representing the empirical probability distribution of $C(T)$ for a fixed time $T$, in two particular cases, $\mu=3$ and $\mu=0.5$. The value of $D$ in each case was adjusted accordingly in order to keep a constant mean. These histograms were obtained by simulating 50, 000 independent concentration paths in each scenario until time $T$, and dividing the outcomes into 100 evenly spaced bins. The time $T$ was chosen large enough so 


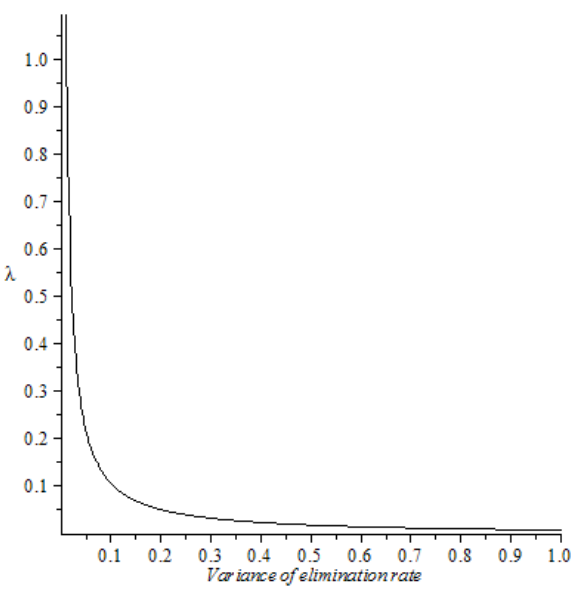

Figure 7: Average number of doses per hour in the non-compliant case as a function of the variance of the elimination rates in a fully compliant population yielding same variance for the concentration (elimination rate $k=0.022 h^{-1}$ ).

that the steady state has been reached (we took $T=100 \max \left(\frac{1}{\lambda}, \frac{1}{k}\right)$ ). The singularity of the distribution of $C$ at the origin in the case $\mu<1$ (second histogram) manifests itself through the sharp spike in the first bin.

\subsection{Continuous model with random intake instants and ran- dom doses}

In this section, we generalize the continuous model of the previous subsection to allow for random doses. The idea is that the careless patient that has an irregular schedule is likely to also mess up with the doses. For instance, he might take a double dose to make up for a missing one (this is a particular case we consider in Subsection 4.1). Formally, this translates into the fact that the quantity $D$ in (8) is now a random variable that may vary at each take rather than being a constant. In other words, we assume now that the concentration, denoted $C^{r d}(t)$, is given by:

$$
C^{r d}(t)=\frac{D}{V_{d}} \sum_{i=0}^{\infty} D_{i} \exp \left(-k_{e}\left(t-T_{i}\right)\right) \mathbb{1}\left(t \geqslant T_{i}\right),
$$

where $D$ and $V_{d}$ are constants, the $\left(T_{i}\right)_{i \in \mathbf{N}}$ again form a Poisson process, and the $\left(D_{i}\right)_{i \in \mathbf{N}}$ are random variables. At time $i$, the dose taken is thus $D D_{i}$. It seems 

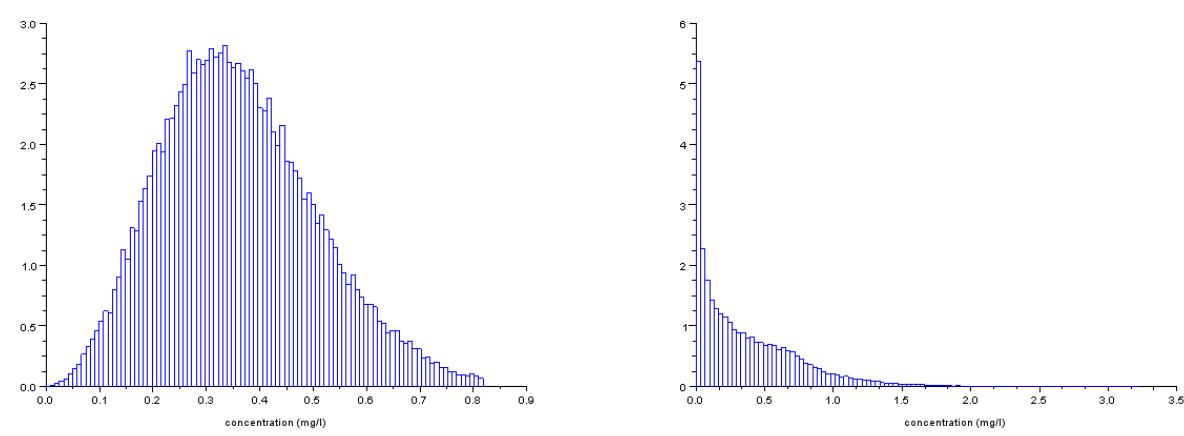

Figure 8: Histogram of the distribution of $C$ (left : $\mu=3$, right : $\mu=0.5$ ).

$$
\begin{array}{|l|l|l|l|l|l|}
\hline a=0.2 & b=1.8 & d_{1}=0.4 & d_{2}=1.9 & q_{1}=0.6 & q_{2}=0.4 \\
\hline
\end{array}
$$

Table 2: Numerical values of the parameters of the random doses distributions used in the simulations.

natural to assume that $\mathbb{E}\left(D_{i}\right)=1$ (i.e on average, the patient takes the required dose), that $D_{i}$ is supported on $\mathbf{R}_{+}$, and that it has compact support, i.e. the patient cannot take arbitrarily large or small doses.

Once again we illustrate this model by showing on Figure 9 simulated sample paths of the concentration in the two particular cases considered in Section 4: in the first one, the random factors $D_{i}$ follow a uniform distribution on the interval $[a, b]$, and, in the second one, they follow a discrete distribution where the $D_{i}$ can take on two values $d_{1}$ and $d_{2}$ with probabilities $q_{1}, q_{2}$. The PK parameters are the ones given in Table 1, with an average time between intakes equal to $\lambda^{-1}=12 \mathrm{~h}$. The parameters for the random doses are given in Table 2.

As shown in Section 4, the mean value in this model is the same as in the previous one, i.e.

$$
\mathbb{E}\left(C^{r d}(t)\right)=\mathbb{E}(C(t)) .
$$

The general expression for the variance is not illuminating (see Formula (25)). However, under the assumption that the variance of $D_{i}$ does not depend on the value of $T_{i}$, one obtains the simple equality:

$$
\operatorname{Var}\left(C^{r d}(t)\right)=\mathbb{E}\left(D_{1}^{2}\right) \operatorname{Var}(C(t))=\left(1+\operatorname{Var}\left(D_{1}\right)\right) \operatorname{Var}(C(t)) .
$$



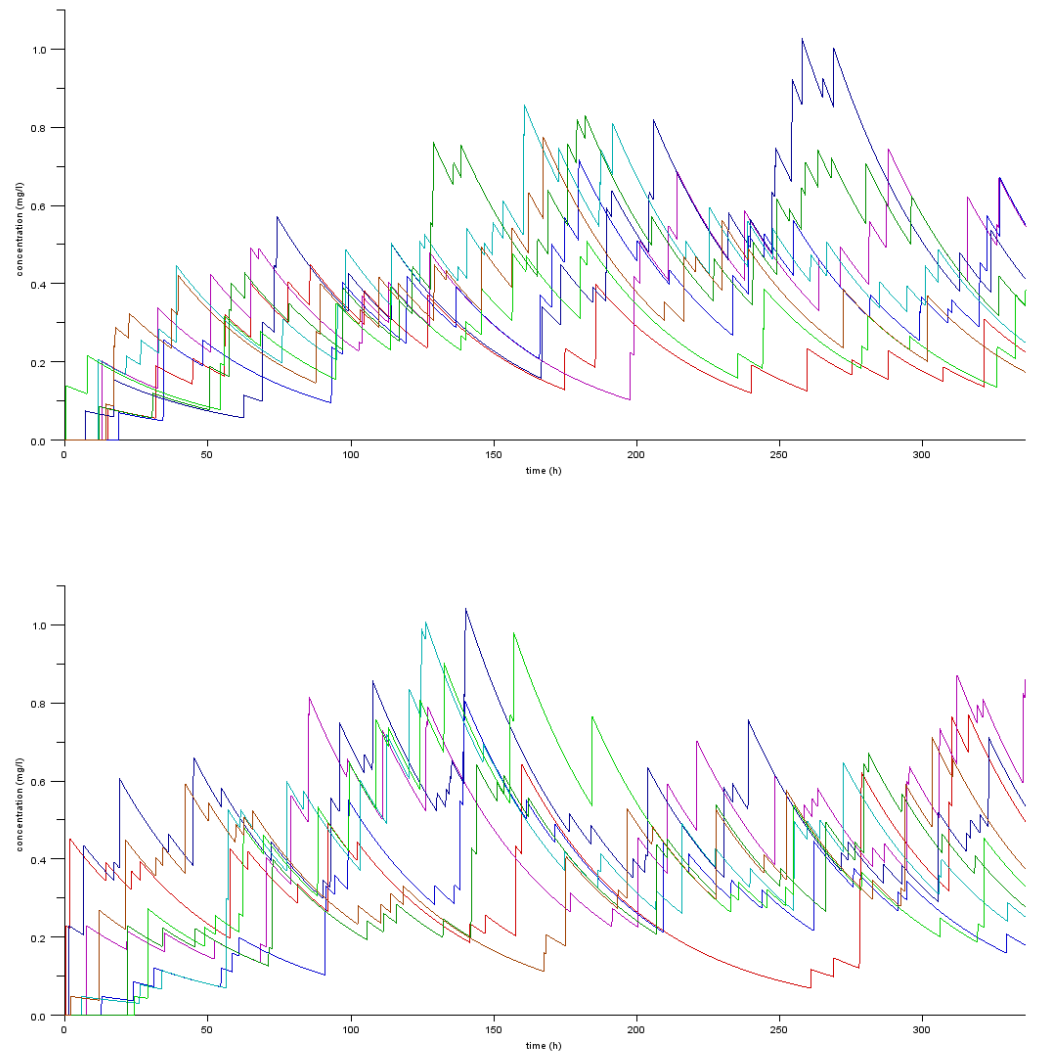

Figure 9: Eight sample paths of the concentration with Poisson distributed intakes and random doses for the first 14 days, in two different scenarios (top: uniform distribution, bottom: discrete distribution). 
Thus, random dosing results in multiplying the variance of the deterministic dose case by $\mathbb{E}\left(D_{1}^{2}\right)$. Estimating quantities such as $\mathbb{P}(|C-\mathbb{E}(C)| \geqslant \gamma \mathbb{E}(C))$ or $\mathbb{P}(C \geqslant$ $U)$, or comparing to the case of (population) full compliance is then readily done once $\operatorname{Var}\left(D_{1}\right)$ is known. In Subsection 4.1, we look at two particular cases where $D_{i}$ is either uniformly distributed on a interval or takes values in a discrete set. We characterize the situations leading to the largest variances for both distributions (Formulas (30) and (31)).

In contrast to variability, random dosing does not affect the regularity of the distribution of the long term concentration; this distribution is again smooth when $\mu \geqslant 1$ and is singular at 0 otherwise.

\subsection{Discrete model with random intake instants and determin- istic doses}

We study a time discretized version of the random model presented in Subsection 2.3. The general idea is that, instead of taking the drug at arbitrary time instants $t \in \mathbf{R}^{+}$, the patient will only do so at times which form a random subset of $\left\{t_{l}^{n}=l / n, l \in \mathbf{N}\right\}$, where $n$ is a fixed number. There are two main reasons for considering such a model. First, there are indeed natural situations where a time discretization does occur. For instance, the medication must sometimes be taken at precise moments, like before lunch. Many people will always have their lunch at a fixed time, like certain workers or many older people. Second, as we show in Subsection 5.3, the time discretized model tends to the continuous one when the discretization step tends to 0 . Thus, for $n$ large enough, the practical difference between both models vanishes. Nonetheless, the discrete model displays various interesting and intriguing features that are not present in the continuous one. Let us finally mention that considering the discretized model is very close to sampling the concentration of the continuous one. Since blood concentrations cannot be monitored continuously, the outcome of any clinical study is discrete in nature, which gives further justification for the discrete model.

We show in Subsection 5.1 that the steady state discretized concentration $S$ in the situation where the patient takes a dose $D$ with probability $p$ independently at each time $j / n$ reads:

$$
S=\frac{D}{V_{d}} \sum_{j=0}^{\infty} a^{j} X_{j},
$$

where the $\left(X_{j}\right)_{j \in \mathbf{N}}$ are i.i.d. Bernoulli random variables with parameter $p$ (i.e. $X_{j}=0$ with probability $1-p$ and $X_{j}=1$ with probability $p$ ). As explained in 
Subsection 5.1, one has to set $p=\frac{\lambda}{n}$ and $a=\exp (-k / n)$ to ensure correspondence between the discrete and continuous models.

The mean and variance of the discretized concentration read:

$$
\mathbb{E}(S)=\frac{D}{V_{d}} \frac{p}{1-a}, \operatorname{Var}(S)=\left(\frac{D}{V_{d}}\right)^{2} \frac{p-p^{2}}{1-a^{2}} .
$$

These formulas allow one to draw the following conclusions:

- for a fixed mean equal to 1 , the variance of $S$ behaves as $\frac{1-p}{p} \frac{1-a}{1+a}$. The parameter $\mu$ is no more relevant here as both $\lambda$ and $k$ play an individual role in this formula. Since we are interested in compliance, we consider the limit cases where $\lambda$ tends to 0 or infinity for a fixed $k$ (and thus a fixed $a$ ). When $\lambda$ tends to 0 , so does $p$, and the variance tends to infinity at speed $\frac{1}{p} \frac{1-a}{1+a}$. This is similar to the continuous model, as $\frac{1}{p}$ is proportional to $\frac{1}{\mu}$. Letting $\lambda$ tend to infinity amounts to letting $p$ tend to 1 , in which case the variance tends to 0 , as expected.

- As above, the bound $\mathbb{P}(|S-\mathbb{E}(S)| \geqslant \gamma \mathbb{E}(S)) \leqslant \frac{1}{\gamma^{2}} \frac{1-p}{p} \frac{1-a}{1+a}$ allows one to assess the effect of non compliance on the variability of the long term concentration.

The study of the regularity of the distribution is much more involved in the discrete case than in the continuous one, as the former may display a fractal behaviour. Indeed, for some values of the parameters, the distribution will be everywhere singular. This means that the evolution of the distribution varies wildly for most values of the concentration. In addition, the dichotomy smooth/singular is not governed by $\mu$ alone, but by complex relations between $\lambda, k$, and the discretization step. More precisely, the cumulative probability distribution is singular either when $\mu \log (2)<p$ or when $p \in\left[\frac{1}{3}, \frac{2}{3}\right]$ and

$$
\frac{k}{n}>-\left[\frac{\lambda}{n} \log \left(\frac{\lambda}{n}\right)+\left(1-\frac{\lambda}{n}\right) \log \left(1-\frac{\lambda}{n}\right)\right] .
$$

It is absolutely continuous when $p \in\left[\frac{1}{3}, \frac{2}{3}\right]$ and

$$
\frac{k}{n}<-\left[\frac{\lambda}{n} \log \left(\frac{\lambda}{n}\right)+\left(1-\frac{\lambda}{n}\right) \log \left(1-\frac{\lambda}{n}\right)\right] .
$$

The situation is not fully understood for $p$ outside the interval $\left[\frac{1}{3}, \frac{2}{3}\right]$. See Subsection 5.4 for more details. 


\subsection{Discrete model with random intake instants and random doses}

Similarly to Subsection 2.4 , we consider a random dose variant of the previous model. The steady state concentration now reads:

$$
S^{r d}=\frac{D}{V_{d}} \sum_{j=0}^{\infty} a^{j} D_{j} X_{j},
$$

which accounts for the fact that the stochastic dose at time $j / n$ is equal to 0 or $D D_{j}$, where the random variables $D_{j}$ are i.i.d. with mean 1 and take values in an interval $\left[d_{m}, d_{M}\right]$ with $0<d_{m}<d_{M}<\infty$. The following facts hold concerning the variability of $S^{r d}$ (see Subsection 6.1):

- the variance of $S^{r d}$ is the one of the discrete model with deterministic dose case above, multiplied by $\left(\frac{\operatorname{Var}\left(D_{1}\right)}{1-p}+1\right)$. It thus has a behaviour similar to the one of $S$. Note however that, for all positive $p$, the effect of random dosing on the variability is amplified in the discrete model, due to the factor $\frac{1}{1-p}$. When $p$ is close to 1 (the number of possible times for intake is only slightly larger than the number of required ones), even a small variability in dosing will have a drastic effect on the variance of the concentration.

- the probability that $S^{r d}$ differs from its mean by a factor $\gamma$ is bounded as follows: $\mathbb{P}\left(\left|S^{r d}-\mathbb{E}\left(S^{r d}\right)\right| \geqslant \gamma \mathbb{E}\left(S^{r d}\right)\right) \leqslant\left(\frac{\operatorname{Var}\left(D_{1}\right)}{1-p}+1\right) \frac{1}{\gamma^{2}} \frac{1-p}{p} \frac{1-a}{1+a}$. The same remarks as above apply.

Concerning regularity, and in contrast to what is observed in the continuous time model, random dosing does affect it. Indeed, when dose randomness is "large", in the sense that $\frac{d_{M}}{d_{m}}$ is sufficiently large, then the distribution of concentration will be singular for most values of $\mu$ and $p$. See Subsection 6.3 for more details, where it is shown that the cumulative probability distribution is singular with a nowhere dense support when $a<\frac{d_{m}}{d_{m}+d_{M}}$. This roughly means that, in addition to the fact that the variations of $S^{r d}$ are very irregular, concentration will only take some particular values in the interval $\left[0, \frac{D d_{M}}{V_{d}} \frac{1}{1-a}\right]$. 


\subsection{Discrete model with random intake instants, random doses, and random elimination rate}

In this section, we consider yet another influence of randomness on the behaviour of the concentration: in addition to random dosing and intake instants, we allow the elimination rate to vary in a stochastic manner in time. While this is not strictly related to the problem of non-compliance, it will obviously has an effect on the variability of the concentration. The rationale for considering this effect is that it seems plausible that, in general, $k$ is not constant in time, and will evolve according to various physiological factors. Of course, over small time intervals, these evolutions will also be small, and are best seen as fluctuations around a mean value. However, as we will see, even small fluctuations have a notable impact on the variability and regularity of the concentration, in particular in the case where $\mu$ is large. We shall restrict to the discrete setting, where a fairly complete description may be obtained.

In details, we consider here a generalization of (11) where the elimination rate at time $j / n$ is equal to $a Z_{j}$ instead of $a$, where the $Z_{j}$ are i.i.d. random variables compactly supported with mean one (so that the mean elimination rate is $a$ ). The asymptotic concentration for large times is denoted $S^{\text {rerd }}$. It reads (see Section 7 for the derivation of this expression):

$$
S^{\text {rerd }}=\frac{D}{V_{d}} \sum_{j=0}^{\infty}\left(\prod_{n=0}^{j-1} Z_{n}\right) a^{j} D_{j} X_{j} .
$$

The study of the variability of $S^{\text {rerd }}$ leads to the following conclusions:

- for a fixed mean equal to 1 , the variance of $S^{\text {rerd }}$ tends to $\frac{\mathbb{E}\left(D_{1}^{2}\right)-p}{p}$ when $\mu \rightarrow$ 0 in the case where $\mathbb{E}\left(Z_{1}^{2}\right)$ remains bounded. However, if, say, $\mathbb{E}\left(Z_{1}^{2}\right) \sim \frac{r}{a^{2}}$ with $0<r<1$, then the variance tends to $\frac{\mathbb{E}\left(D_{1}^{2}\right)-p(1-r)}{p(1-r)}$. The situation when $\mu \rightarrow \infty$ depends more drastically on the behaviour of the random elimination rate: when $\mu$ tends to infinity, the $Z_{i}$ must tend almost surely to 1. If for instance $\mathbb{E}\left(Z_{1}^{2}\right) \sim\left(\frac{1}{a^{2}}\right)^{r}$ again with $0<r<1$, then the variance tends to $\frac{1}{1-r}$. However, if $\mathbb{E}\left(Z_{1}^{2}\right) \sim 1+\frac{2 p}{\mu}$, then the variance tends to infinity as $\frac{\mu}{p}$ (the variance will also tend to infinity if $\mathbb{E}\left(Z_{1}^{2}\right)$ tends to 1 more slowly that $1+\frac{2 p}{\mu}$ but remains smaller than $\frac{1}{a^{2}}$ ). Thus, as soon as elimination varies randomly in time, the variance of the concentration does not tend to 0 when $\mu$ tends to infinity in this model, as it does for all other models considered in this work. It may even increase without bounds in certain situations; 
- again, bounds for $\mathbb{P}\left(\left|S^{\text {rerd }}-\mathbb{E}\left(S^{\text {rerd }}\right)\right| \geqslant \gamma \mathbb{E}\left(S^{\text {rerd }}\right)\right)$ are given by the analysis of the variance above: when $\mu \rightarrow 0$, we get a finite bound equal to $\frac{\mathbb{E}\left(D_{1}^{2}\right)-p}{p}$ or a similar term depending on the behaviour of the elimination rate. Contrarily to all other models, when $\mu$ tends to infinity, the bound does not tend to 0 , which means that even for closely spaced intake instants, a strong variability of the concentration will remain.

Using Proposition 11 of Subection 7.1, we plot on Figure 10 the variance of the concentration as a function of the variances of the random elimination rate and the random dose. As one can see, the added variability due to random elimination has far greater impact on the variance of the concentration than the one due to random dosing. Thus, "small" fluctuations in time of the elimination rate have more impact than "large" fluctuations in dosing.

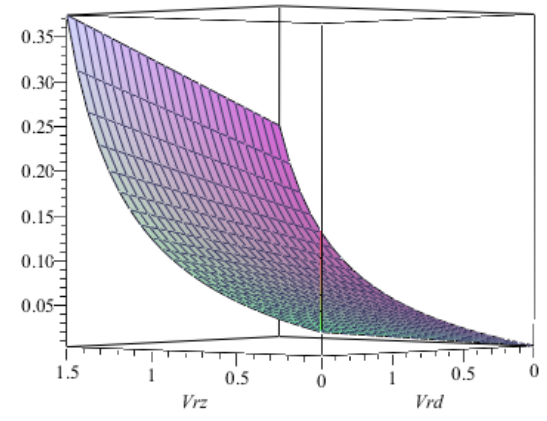

Figure 10: Variance of the concentration as a function of the variances of the random elimination (Vrz) and the random dosing (Vrd), with values as in Table 1, $p=0.8,1 / n=24$ hours (and thus $a=0.59$ ).

Let us now turn to the regularity of the concentration: in most situations, it will be irregular, except for moderate values of $p$ and large $\mu$. More precisely, the cumulative probability distribution is singular when

$$
\mathbb{E}(\log (Z))<p / \mu+p \log (p)+(1-p) \log (1-p)
$$

and it is absolutely continuous when the reverse inequality holds. In other words, singularity will occur frequently when rate elimination randomness is "large", in 
the sense that the logarithmic moment of the distribution of $Z$ is sufficiently large in absolute value. See Subsection 7.2 for more details.

This ends the overview of our models and their main features. The reader will find in the next sections the precise derivations of the results mentioned above, along with a more detailed analysis of the models.

\section{Continuous model with random instants and de- terministic doses}

We study in this section the main properties of the random concentration given by (8), that we recall here for convenience:

$$
C(t)=\frac{D}{V_{d}} \sum_{i=0}^{\infty} \exp \left(-k\left(t-T_{i}\right)\right) \mathbb{1}\left(t \geqslant T_{i}\right),
$$

where the $T_{i}$ are Poisson distributed with parameter $\lambda$. Let us compute the characteristic function $\varphi_{t}$ of $C(t)$. One version of Campbell theorem [8] states that the process $\sum_{X \in \Pi} f(X)$, where $\Pi$ is a Poisson process on a measurable subset $S$ of $\mathbf{R}$ with mean measure $\nu$ and $f$ a real-valued measurable function, is absolutely convergent if and only if $\int_{S} \min (1,|f(x)|) \nu(d x)<\infty$, in which case its characteristic function equals $\exp \left(\int_{S}\left(e^{i \theta f(x)}-1\right) \nu(d x)\right)$. Applying this result to $S=\mathbf{R}^{+}, \nu(d x)=\lambda d x$ and $f(x)=\frac{D}{V_{d}} \exp (-k(t-x)) \mathbb{1}(t \geqslant x)$, one gets:

$$
\varphi_{t}(\theta)=\exp \left[\lambda \int_{0}^{t}\left(\exp \left(i \theta \frac{D}{V_{d}} e^{-k(t-x)}\right)-1\right) d x\right] .
$$

The change of variable $u=\frac{D}{V_{d}} e^{-k(t-x)}$ yields:

Proposition 1. The characteristic function of $C(t)$ is:

$$
\varphi_{t}(\theta)=\exp \left(\mu \int_{\theta \frac{D}{V_{d}} e^{-k t}}^{\theta \frac{D}{V_{d}}} \frac{e^{i u}-1}{u} d u\right)
$$

where $\mu=\frac{\lambda}{k}$.

The parameter $\mu$ is the inverse of the "oscillating parameter" considered in [9]. Similarly to [9], we see that it plays a major role in the analysis of random compliance. 


\subsection{Variability of the concentration}

From the characteristic function, one easily gets:

$$
\mathbb{E}(C(t))=\mu \frac{D}{V_{d}}\left(1-e^{-k t}\right)
$$

so that:

$$
\mathbb{E}(C(t)) \rightarrow_{t \rightarrow \infty} \mu \frac{D}{V_{d}}
$$

Likewise, the variance of $C(t)$ reads:

$$
\operatorname{Var}(C(t))=\frac{\mu}{2}\left(\frac{D}{V_{d}}\right)^{2}\left(1-e^{-2 k t}\right) .
$$

When $t$ tends to infinity,

$$
\operatorname{Var}(C(t)) \rightarrow_{t \rightarrow \infty} \frac{\mu}{2}\left(\frac{D}{V_{d}}\right)^{2} .
$$

Note that the convergences in (12) and (13) are exponential: only a few cycles are needed before the steady state is reached. The same remark applies to all convergences below.

In the next section, we show that, when $t$ tends to infinity, $C(t)$ itself also converges to a well-defined random variable, denoted $C$, and investigate in details some of its properties. Before that, we give a final result of interest pertaining to the variability of the concentration in the non-compliant case. More precisely, the following proposition yields a bound on the probability that the concentration exceeds a given (large) level, or departs significantly from its mean.

\section{Proposition 2.}

$$
\begin{gathered}
\mathbb{P}(C(t) \geqslant U) \leqslant \frac{7}{12 U^{2}} \mu\left(\frac{D}{V_{d}}\right)^{2}\left(1-e^{-k t}\right)\left(1+2 \mu-2 \mu e^{-k t}+e^{-k t}\right) . \\
\mathbb{P}(C \geqslant U) \leqslant \frac{7}{6 U^{2}}\left(\frac{D}{V_{d}}\right)^{2} \mu\left(\mu+\frac{1}{2}\right) . \\
\mathbb{P}(|C-\mathbb{E}(C)| \geqslant \gamma \mathbb{E}(C)) \leqslant \frac{1}{2 \mu \gamma^{2}} .
\end{gathered}
$$


Of course, the bound in (16) is of interest only if $\mu$ and/or $\gamma$ is sufficiently large.

Proof.

This is a direct application of the classical bound (see, e.g. [11], p. 209):

$$
\mathbb{P}(C(t) \geqslant U) \leqslant 7 U \int_{0}^{\frac{1}{U}}\left(1-\operatorname{Re}\left(\varphi_{t}(\theta)\right)\right) d \theta,
$$

valid for $U>0$, and where Re denotes the real part. Indeed,

$$
\operatorname{Re}\left(\varphi_{t}(\theta)\right)=\cos \left(\mu \int_{\theta \frac{D}{V_{d}} e^{-k t}}^{\theta \frac{D}{V_{d}}} \frac{\sin (u)}{u} d u\right) \exp \left(\mu \int_{0}^{\theta \frac{D}{V_{d}}} \frac{\cos (u)-1}{u} d u\right)
$$

and routine estimates yield (14). (15) follows in a similar way. Finally, (16) is simply Chebychev inequality.

Note that, in the deterministic case (full compliance), and with the definition of the variance given in Subsection 2.1, one has, in place of (16):

$$
\mathbb{P}\left(\left|C_{d}(t)-\mathbb{E}\left(C_{d}\right)\right| \geqslant \gamma \mathbb{E}\left(C_{d}\right)\right) \leqslant \frac{1}{2 \mu \gamma^{2}} \frac{e^{\frac{1}{\mu}}-2 \mu e^{\frac{1}{\mu}}+2 \mu+1}{e^{\frac{1}{\mu}}-1} .
$$

This is another quantitative way to measure by how much the probability of differing from the mean will be larger in the non-compliant case: in particular, when $\mu$ is "large", the right-hand side in the inequality above behaves as $\frac{1}{12 \mu^{2} \gamma^{2}}$. This gives a bound six times smaller than in the non-compliant case. This was illustrated on Figure 3.

\subsection{Regularity of the limit distribution}

In this section, we study the long term behaviour of the drug concentration, i.e. the distribution function of the $\operatorname{limit} C:=\lim _{t \rightarrow \infty} C(t)$, where the limit is understood to be taken in the sense of convergence in law. Indeed:

Proposition 3. The random variables $C(t)$ converge in distribution, when tends to infinity, to a well defined random variable $C$ whose characteristic function equals:

$$
\varphi(\theta)=\exp \left(\mu \int_{0}^{\theta \frac{D}{V_{d}}} \frac{e^{i u}-1}{u} d u\right) .
$$


Proof.

When $t$ tends to infinity, $\varphi_{t}$ tends pointwise to $\varphi$, which is continuous at $\theta=0$. By Levy theorem, this implies the result.

We shall denote by $F$ the cumulative probability distribution of $C$, i.e. $F(x)=$ $\mathbb{P}(C \leqslant x)$. It is convenient to have an explicit expression for $C$. However, one cannot directly let $t$ tend to infinity in (1) to obtain a representation of the limit concentration. One way around this difficulty is to notice that the distribution of the intake times falling in the interval $[0, t]$ is invariant by time reversal: looking "backwards" in time, one sees that $C(t)$ has the same law as $\left.C^{\prime}(t)=\frac{D}{V_{d}} \sum_{i=0}^{\infty} \exp \left(-k T_{i}\right)\right) \mathbb{1}\left(t \geqslant T_{i}\right)$; as $t$ tends to infinity, the random variables $C^{\prime}(t)$ converge almost surely to $C^{\prime}=\frac{D}{V_{d}} \sum_{i=0}^{\infty} \exp \left(-k_{e} T_{i}\right)$, which therefore has the same distribution as $C$.

In the sequel, we shall write

$$
C=\frac{D}{V_{d}} \sum_{i=0}^{\infty} \exp \left(-k T_{i}\right)
$$

since we are only interested in distributional properties.

One may rewrite $\varphi$ as follows:

$$
\varphi(\theta)=\left(\frac{D}{V_{d}}\right)^{-\mu} e^{-\mu \gamma} e^{-\frac{i \mu \pi}{2}} \theta^{-\mu} \exp \left(-\mu \int_{1}^{\infty} \frac{e^{i \frac{D}{V_{d}}} \theta u}{u} d u\right)
$$

where $\gamma$ is Euler's constant.

Proposition 4. When $\theta$ tends to infinity,

$$
\varphi(\theta) \sim\left(\frac{D}{V_{d}}\right)^{-\mu} e^{-\mu \gamma+i \mu \frac{\pi}{2}} \theta^{-\mu}+O\left(\theta^{-\mu-1}\right) .
$$

Proof.

When $\theta$ tends to infinity, $\int_{0}^{\frac{D}{V_{d}} \theta} \frac{\sin (u)}{u} d u$ tends to $\int_{0}^{\infty} \frac{\sin (u)}{u} d u=\frac{\pi}{2}$, while:

$$
\int_{0}^{\frac{D}{V_{d}} \theta} \frac{\cos (u)-1}{u} d u \sim-\gamma-\log \left(\frac{D}{V_{d}} \theta\right)+O\left(\frac{1}{\theta}\right) .
$$


In particular, $\int_{0}^{\frac{D}{V_{d}} \theta} \frac{e^{i u}-1}{u} d u-i \frac{\pi}{2}+\gamma+\log \left(\frac{D}{V_{d}} \theta\right)$ tends to 0 and thus we may exponentiate the equivalents to get the announced result.

In particular,

$$
|\varphi(\theta)| \sim K \theta^{-\mu}, \quad \theta \rightarrow \infty
$$

where $K$ is a constant (in the sequel, $K$ will always denote a finite positive constant, which may change from line to line).

From this, we get that $F$ has an $L^{2}$ density $^{2}$ if and only if $\mu>1 / 2$.

Recall the following well-known properties (see, e.g. [16], p. 40): let $\alpha \in$ $(0,1)$. Then:

1. $\frac{1}{T} \int_{-T}^{T}|\varphi(\theta)|^{2} d \theta=O\left(T^{-\alpha}\right) \Rightarrow F \in \operatorname{Lip}\left(\frac{\alpha}{2}\right)$,

2. $\int_{1}^{\infty} \theta^{\alpha-1}|\varphi(\theta)| d \theta<\infty \Rightarrow F \in \operatorname{Lip}(\alpha)$.

From 2 and (21), one gets $F \in \operatorname{Lip}(\mu-\varepsilon)$ for $\mu<1$ and any $\varepsilon>0$. One easily verifies that $\frac{1}{T} \int_{-T}^{T}|\varphi(\theta)|^{2} d \theta$ is equivalent to $K T^{-2 \mu}$ when $\mu<\frac{1}{2}$ and to $K T^{-1}$ when $\mu>\frac{1}{2}$. From this and 1 , one deduces that $F \in \operatorname{Lip}(\mu)$ for $\mu<\frac{1}{2}$.

A more localized information on the regularity of $F$ may be obtained from its Laplace transform $\psi(u)$, whose expression also follows from Campbell theorem (since $C$ is positive, the conditions of Campbell theorem are verified):

$$
\psi(u)=\exp \left[\mu \int_{0}^{u \frac{D}{V_{d}}}\left(\frac{e^{-s}-1}{s}\right) d s\right] .
$$

It is facile to deduce from this that $\psi(u) \sim e^{-\mu \gamma-\mu \log \left(\frac{D}{V_{d}}\right)} u^{-\mu}$ when $u$ tends to infinity. A classical Tauberian theorem (see, e.g. [5], p.445) then entails that:

$$
F(\varepsilon) \sim \frac{e^{-\mu \gamma-\mu \log \left(\frac{D}{V_{d}}\right)}}{\Gamma(\mu+1)} \varepsilon^{\mu}, \quad \varepsilon \rightarrow 0 .
$$

Thus, $F$ has a finite non vanishing derivative at 0 exactly when $\mu=1$. Furthermore, it is differentiable at 0 when $\mu \geqslant 1$ and not differentiable at 0 when $\mu<1$. In addition, 0 is the only possibly singular point of $F$. Indeed, the following proposition shows that $F$ is smooth at any $x>0$.

\footnotetext{
${ }^{2}$ This means that the integral of the square of the probability density function is finite.
} 
Proposition 5. For any $x>0$,

$$
F(x+\varepsilon)-F(x)=O(\varepsilon)
$$

when $\varepsilon \rightarrow 0^{+}$.

The proof is given in section 8.1.

The practical meaning of these results is that, exactly when $\lambda<k$, the instants of intakes are too scarce (with respect to the elimination rate), resulting in a high probability of having too small a concentration of drugs.

\section{Continuous model with random instants and ran- dom doses}

In this section, we consider the generalization of the continuous model of Section 3 to allow for random doses. The concentration, denoted $C^{r d}(t)$, now reads:

$$
C^{r d}(t)=\frac{D}{V_{d}} \sum_{i=0}^{\infty} D_{i} \exp \left(-k_{e}\left(t-T_{i}\right)\right) \mathbb{1}\left(t \geqslant T_{i}\right)
$$

where the $\left(T_{i}\right)_{i \in \mathbf{N}}$ form again a Poisson process, and the $\left(D_{i}\right)_{i \in \mathbf{N}}$ are random variables. At time $T_{i}$, the dose taken is $D D_{i}$ and we assume that $\mathbb{E}\left(D_{i}\right)=1$, that $D_{i}$ is supported on $\mathbf{R}_{+}$, and that it has compact support (although we shall need only weaker assumptions).

The process $C^{r d}(t)$ thus defined is a marked Poisson process. In this work, we shall assume that the $\left(D_{i}\right)_{i \in \mathbf{N}}$ are independent and identically distributed random variables, where each $D_{i}$ may depend on $T_{i}$ but is independent from the $\left(T_{j}\right)_{j \neq i}$. This makes sense from a pharmacokinetical point of view, since it seems plausible that the patient will not adjust his dose at time $T_{i}$ on the basis of his past or future behaviour except for the time lag from the previous take, although it would maybe be desirable to let $D_{i}$ depend also on $D_{i-1}$. We denote by $\nu(T,$.$) the conditional$ distribution of $D_{i}$ knowing that $T_{i}=T$. Our assumptions makes it possible to apply a generalized form of Campbell theorem [8] to the effect that the characteristic function $\varphi_{t}^{r d}$ of $C^{r d}(t)$ is given by:

$$
\varphi_{t}^{r d}(\theta)=\exp \left\{\lambda \int_{\mathbf{R}_{+} \times A}\left[\exp \left(i \theta \frac{D}{V_{d}} u e^{-k(t-x)} \mathbb{1}(t \geqslant x)\right)-1\right] \nu(x, d u) d x\right\}
$$




$$
=\exp \left\{\lambda \int_{0}^{t} \int_{A}\left[\exp \left(i \theta \frac{D}{V_{d}} u e^{-k(t-x)}\right)-1\right] \nu(x, d u) d x\right\},
$$

where $A \subset \mathbf{R}_{+}^{*}$ is the support of the $D_{i}$.

\subsection{Variability of the concentration}

From (23), one deduces easily the mean and variance of $C^{r d}$ :

$$
\left(\varphi_{t}^{r d}\right)^{\prime}(\theta)=\varphi_{t}^{r d}(\theta) \lambda \int_{0}^{t} \int_{A} i \frac{D}{V_{d}} u e^{-k(t-x)} \exp \left(i \theta \frac{D}{V_{d}} u e^{-k(t-x)}\right) \nu(x, d u) d x
$$

and thus

$$
\begin{aligned}
\left(\varphi_{t}^{r d}\right)^{\prime}(0) & =i \lambda \frac{D}{V_{d}} \int_{0}^{t} e^{-k(t-x)} \int_{A} u \nu(x, d u) d x \\
& =i \lambda \frac{D}{V_{d}} \int_{0}^{t} e^{-k(t-x)} \mathbb{E}_{x}\left(D_{1}\right) d x \\
& =i \lambda \frac{D}{V_{d}} \int_{0}^{t} e^{-k(t-x)} d x \\
& =i \mu \frac{D}{V_{d}}\left(1-e^{-k t}\right)
\end{aligned}
$$

where $\mathbb{E}_{x}\left(D_{1}\right)$ denotes the expectation of $D_{i}$ knowing that $T_{i}=x$, which is equal to one for any $x$ by assumption. Thus we find that, not surprisingly:

$$
\mathbb{E}\left(C^{r d}(t)\right)=\mathbb{E}(C(t)) .
$$

Likewise,

$$
\begin{aligned}
\left(\varphi_{t}^{r d}\right)^{\prime \prime}(\theta)= & \varphi_{t}^{r d}(\theta) \lambda^{2}\left(\int_{0}^{t} \int_{A} i \frac{D}{V_{d}} u e^{-k(t-x)} \exp \left(i \theta \frac{D}{V_{d}} u e^{-k(t-x)}\right) \nu(x, d u) d x\right)^{2}+ \\
& \varphi_{t}^{r d}(\theta) \lambda \int_{0}^{t} \int_{A}-\left(\frac{D}{V_{d}}\right)^{2} u^{2} e^{-2 k(t-x)} \exp \left(i \theta \frac{D}{V_{d}} u e^{-k(t-x)}\right) \nu(x, d u) d x
\end{aligned}
$$

Using that, by definition, $\int_{A} u^{2} \nu(x, d u)=\mathbb{E}_{x}\left(D_{1}^{2}\right)$, this entails:

$$
\begin{aligned}
\left(\varphi_{t}^{r d}\right)^{\prime \prime}(0) & =-\mathbb{E}(C(t))^{2}-\lambda\left(\frac{D}{V_{d}}\right)^{2} \int_{0}^{t} e^{-2 k(t-x)} \int_{A} u^{2} \nu(x, d u) d x \\
& =-\mathbb{E}(C(t))^{2}-\lambda\left(\frac{D}{V_{d}}\right)^{2} \int_{0}^{t} e^{-2 k(t-x)} \mathbb{E}_{x}\left(D_{1}^{2}\right) d x
\end{aligned}
$$


or, since $\left(\varphi_{t}^{r d}\right)^{\prime \prime}(0)=-\mathbb{E}(C(t))^{2}-\operatorname{Var}\left(C^{r d}(t)\right)$,

$$
\operatorname{Var}\left(C^{r d}(t)\right)=\lambda\left(\frac{D}{V_{d}}\right)^{2} \int_{0}^{t} e^{-2 k(t-x)} \mathbb{E}_{x}\left(D_{1}^{2}\right) d x .
$$

However,

$$
\mathbb{E}_{x}\left(D_{1}^{2}\right)=\operatorname{Var}_{x}\left(D_{1}\right)+\mathbb{E}_{x}\left(D_{1}\right)^{2}=\operatorname{Var}_{x}\left(D_{1}\right)+1
$$

$\left(\operatorname{Var}_{x}\left(D_{1}\right)\right.$ denotes the variance of $D_{i}$ knowing that $T_{i}=x$ ) and

$$
\lambda\left(\frac{D}{V_{d}}\right)^{2} \int_{0}^{t} e^{-2 k(t-x)} d x=\operatorname{Var}(C(t))
$$

thus

$$
\operatorname{Var}\left(C^{r d}(t)\right)=\operatorname{Var}(C(t))+\lambda\left(\frac{D}{V_{d}}\right)^{2} \int_{0}^{t} e^{-2 k(t-x)} \operatorname{Var}_{x}\left(D_{1}\right) d x
$$

If we assume that the variance of $D_{i}$ does not depend on the value of $T_{i}$, i.e. $\operatorname{Var}_{x}\left(D_{1}\right)=\operatorname{Var}\left(D_{1}\right)$, we get:

$$
\operatorname{Var}\left(C^{r d}(t)\right)=\mathbb{E}\left(D_{1}^{2}\right) \operatorname{Var}(C(t))=\left(1+\operatorname{Var}\left(D_{1}\right)\right) \operatorname{Var}(C(t)) .
$$

Thus, random dosing results in multiplying the variance of the deterministic dose case by $\mathbb{E}\left(D_{1}^{2}\right)$. It is then easy to obtain inequalities similar to (14), (15), and (16) for the random dose case.

Two particular cases may be of special interest:

- Discrete distribution: the $\left(D_{i}\right)$ assume the values $\left\{d_{1}, \ldots, d_{m}\right\} \subset \mathbf{R}_{+}$with probabilities $q_{1}, \ldots, q_{m}$, independently of the $T_{i}$. We denote by $C_{t}^{r d, d}$ and $\varphi_{t}^{r d, d}$ the concentration and characteristic functions. Then:

$$
\varphi_{t}^{r d, d}(\theta)=\prod_{j=1}^{m} \exp \left(q_{j} \mu \int_{\theta \frac{D}{V_{d}} d_{j} e^{-k t}}^{\theta \frac{D}{V_{d}} d_{j}} \frac{e^{i u}-1}{u} d u\right)
$$

which has an obvious interpretation and may be written as:

$$
\varphi_{t}^{r d, d}(\theta)=\prod_{j=1}^{m} \varphi_{t}\left(q_{j} \mu, d_{j} \theta\right)
$$

where we have written $\varphi_{t}\left(q_{j} \mu,.\right)$ instead of $\varphi_{t}($.$) for the characteristic func-$ tion in the deterministic-dose case but with parameter $q_{j} \mu$ instead of $\mu$. This 
equality also holds when $t$ tends to infinity, yielding the long term concentration characteristic function. The variance is given by:

$$
\operatorname{Var}\left(C^{r d, d}(t)\right)=\mathbb{E}\left(D_{1}^{2}\right) \operatorname{Var}(C(t))=\left(\sum_{j=1}^{m} q_{j} d_{j}^{2}\right) \operatorname{Var}(C(t)) .
$$

It is of interest to characterize the situation giving the largest variance among all admissible random dosings with arbitrary $m, q_{i}$, and $d_{i}$. In other words, we look for the value of $V_{\max }:=\max \operatorname{Var}\left(C^{r d, d}(t)\right)$ subject to $m>1$, $\left(q_{1}, \ldots, q_{m}\right) \in[0,1]^{m}$ with at least two $q_{i}$ non zero and $\sum_{j=1}^{m} q_{i}=1$, $\left(d_{1}, \ldots, d_{m}\right) \in[a, b]$ with $b>a>0$, and $\sum_{j=1}^{m} q_{i} d_{i}=1$. It is easily shown, for instance using Lagrange multipliers, that the maximum is reached for $m=2$ and $d_{1}=a, d_{2}=b$. In this case,

$$
V_{\max }=(a+b-a b) \operatorname{Var}(C(t)) .
$$

Thus the worst-case situation is when the patient "oscillates" between two dosings whose average is the prescribed one.

- Uniform distribution: the $D_{i}$ are uniformly distributed over $[a, b] \subset \mathbf{R}_{+}$, independently of the $T_{i}$. We denote by $C_{t}^{r d, u}$ and $\varphi_{t}^{r d, u}$ the concentration and characteristic function. In this case, one computes:

$$
\begin{aligned}
\varphi_{t}^{r d, u}(\theta) & =\exp \left\{\frac{\lambda}{b-a} \int_{0}^{t} \int_{a}^{b}\left[\exp \left(i \theta \frac{D}{V_{d}} u e^{-k(t-x)}\right)-1\right] d u d x\right\} \\
& =\exp \left\{-\frac{i \mu}{b-a} \int_{\theta \frac{D}{V_{d}} e^{-k t}}^{\theta \frac{D}{V_{d}}}\left[\frac{e^{i b v}-e^{i a v}}{v^{2}}-i \frac{b-a}{v}\right] d v\right\},
\end{aligned}
$$

This last integral is easily seen to be convergent when $t$ tends to infinity, which gives us the long term concentration characteristic function. Note that $\varphi^{r d, u}$ tends to $\varphi$ when the couple $(a, b)$ tends to $(D, D)$ : the concentration with random doses uniformly distributed on $[a, b]$ tends in law to the concentration with fixed dose $D$.

The variance is given by:

$$
\operatorname{Var}\left(C^{u}(t)\right)=\mathbb{E}\left(D_{1}^{2}\right) \operatorname{Var}(C(t))=\frac{a^{2}+a b+b^{2}}{3} \operatorname{Var}(C(t)) .
$$

From this equation it is easily seen that the choice of $[a, b]$ maximizing the variance under the constraint $\mathbb{E}\left(D_{i}\right)=1$ is $a=0, b=2$, as expected. 


\subsection{Regularity of the limit distribution}

We first consider the two cases of discrete and uniform random dosing.

- Discrete distribution

From (28), one easily sees that:

$$
\left|\varphi_{t}^{r d, d}(\theta)\right| \sim \prod_{j=1}^{m}\left(d_{j} \theta\right)^{-q_{j} \mu}=K \theta^{-\mu}
$$

when $\theta$ tends to infinity, where $K$ is a constant.

- Uniform distribution

The modulus of $\varphi^{r d, u}(\theta)=\lim _{t \rightarrow \infty} \varphi_{t}^{r d, u}(\theta)$ equals:

$$
\begin{aligned}
\left|\varphi^{r d, u}(\theta)\right| & =\exp \left\{\frac{\mu}{b-a} \int_{0}^{\theta \frac{D}{V_{d}}}\left[\frac{\sin (b v)-\sin (a v)}{v^{2}}-\frac{b-a}{v}\right] d v\right\} \\
& =\exp \left\{\frac{\mu}{b-a} \int_{0}^{\theta \frac{D}{V_{d}}}\left[\frac{\sin (b v)}{v^{2}}-\frac{b}{v}\right] d v\right\} \\
& \times \exp \left\{\frac{\mu}{b-a} \int_{0}^{\theta \frac{D}{V_{d}}}\left[-\frac{\sin (a v)}{v^{2}}+\frac{a}{v}\right] d v\right\} .
\end{aligned}
$$

Now, for large enough $\theta$,

$$
\begin{aligned}
& \exp \left\{\frac{\mu}{b-a} \int_{0}^{\theta \frac{D}{V_{d}}}\left[\frac{\sin (b v)}{v^{2}}-\frac{b}{v}\right] d v\right\}=\exp \left\{\frac{\mu}{b-a} \int_{0}^{1}\left[\frac{\sin (b v)}{v^{2}}-\frac{b}{v}\right] d v\right\} \\
& \quad \times \exp \left\{\frac{\mu}{b-a} \int_{1}^{\theta \frac{D}{V_{d}}} \frac{\sin (b v)}{v^{2}} d v\right\} \exp \left\{-\frac{\mu}{b-a} \int_{1}^{\theta \frac{D}{V_{d}}} \frac{b}{v} d v\right\} .
\end{aligned}
$$

The first factor of the product in the right-hand side above is a constant. When $\theta$ tends to infinity, the second factor tends to another constant. Finally, the third factor behaves as a constant times $\theta^{-\mu \frac{b}{b-a}}$. As consequence,

$$
\left|\varphi^{r d, u}(\theta)\right| \sim K \theta^{-\mu \frac{b}{b-a}} \theta^{\mu \frac{a}{b-a}}=K \theta^{-\mu}
$$

when $\theta$ tends to infinity. 
Thus in both particular cases above, we recover the same behaviour for the characteristic function as in the deterministic-dose case. As the next proposition shows, this is in fact a general feature of all random dosings provided $\nu(x, d u)$ does not depend on $x$, with an additional mild condition:

Proposition 6. Assume that $\nu(x, d u)=\nu(d u)$ and that $\int_{A} \log (u) \nu(d u)<\infty$. Then

$$
\varphi^{r d}(\theta) \sim K \theta^{-\mu}
$$

when $\theta$ tends to infinity, where $K \in \mathrm{C}$ is a constant.

The proof of this proposition may be found in Section 8.2

Proposition 6 shows that random dosing, at least when the distribution of the $D_{i}$ is independent of the $T_{i}$, does not alter the regularity of the distribution of the long term concentration as compared to the deterministic dose case.

\section{Discrete model with random instants and deter- ministic doses}

We study in this section a discretization in time of the model of Section 3: instead of taking the drug at arbitrary time instants $t \in \mathbf{R}^{+}$, we assume that the patient will only do so at times which form a random subset of $\left\{t_{l}^{n}=l / n, l \in \mathbf{N}\right\}$, where $n$ is a fixed number.

We shall first rewrite the drug concentration in this discrete setting. It will appear that the discretized concentration $C_{d}$ has the same law as a classical measure called infinite Bernoulli convolution. We will study the variability of the discretized concentration. Then we will show that, when $n$ tends to infinity, the discretized model tends distribution-wise to the continuous time one. We will finally study the regularity of the long term behaviour of the discretized concentration for $n$ fixed or tending to infinity, and show that it is, under certain circumstances, singular.

\subsection{The discretized concentration}

Let us explain in detail how to discretize the continuous model of Section 3 in a meaningful way. Let $h=\frac{1}{n}>0$ be the discretization step. We assume that the intakes can only occur at times $t_{j}=j h, j \in \mathbf{N}$. Let $X_{j}$ be the random variable 
that takes on the value 1 if the patient takes the drug at time $t_{j}$, and 0 otherwise.

From a general point of view, the discrete analogue of the Poisson process is the Bernoulli process. Indeed, in the continuous framework, the Poisson process is the only counting process which has stationary and independent increments. The only discrete counting process with the same property is the Bernoulli process. In terms of waiting times, this amounts to replacing the i.i.d., memoryless, exponential random variables $S_{n}=T_{n+1}-T_{n}$, by i.i.d. random variables following a geometric distribution (recall that the geometric distribution is the only memoryless discrete distribution $)^{3}$.

We are thus led to consider a sequence of i.i.d. Bernoulli random variables $\left(X_{j}\right)_{j \geqslant 1}$ as follows:

$$
\begin{aligned}
& X_{j}=0 \text { with probability } 1-p \\
& X_{j}=1 \text { with probability } p .
\end{aligned}
$$

In the discrete model, the expected number of intakes per unit time is $p / h$, so in order to agree with the continuous model, one has to choose $p=\lambda h$. Note that, for the model to make sense, $p$ cannot be larger than one, which translates into:

$$
\lambda h \leqslant 1 .
$$

This simply means that if the required number of doses per month is, say, 10, then there must be least 10 discrete times in the month when intake is possible. The closer $p$ is to 1 , the more constrained the situation is. We shall see various instances of this below, most notably in section 6.1.

At a fixed time $t_{n}$ and for any $0 \leqslant j \leqslant n$, the contribution of the $j$-th intake to the current concentration is

$$
\frac{D}{V_{d}} \exp \left(-k\left(t_{n}-t_{j}\right)\right) X_{j}=\frac{D}{V_{d}} a^{n-j} X_{j}
$$

where $a=\exp (-k h)=\exp (-p / \mu)$ is the elimination rate for one time step. Therefore the total concentration at time $t_{n}$ is given by

$$
C_{n}=\frac{D}{V_{d}} \sum_{j=0}^{n} a^{n-j} X_{j}
$$

\footnotetext{
${ }^{3}$ One may also argue by considering what happens in time intervals of size $h$ : let $X_{j}$ be the number of time instants where a drug intake takes place in such an interval (in the continuous model). For small $h, X_{j}$ is approximately distributed as a Bernoulli random variable.
} 
(Note that the sequence $\left(C_{n}\right)$ satisfies the natural recurrence equation $C_{n+1}=$ $a C_{n}+X_{n+1}$ which shows that $\left(C_{n}\right)$ is a Markov chain on $\mathbf{R}_{+}$.)

Since the random variables $X_{0}, \ldots, X_{n}$ are independent, they are exchangeable, and in particular the vector $\left(X_{0}, \ldots, X_{n}\right)$ has the same distribution as the reversed vector $\left(X_{n}, \ldots, X_{0}\right)$. Hence $C_{n}$ is equal in distribution to

$$
S_{n}=\frac{D}{V_{d}} \sum_{j=0}^{n} a^{j} X_{j} .
$$

The sequence $\left(S_{n}\right)$ converges almost surely to the random variable

$$
S=\frac{D}{V_{d}} \sum_{j=0}^{\infty} a^{j} X_{j} .
$$

This implies that $C_{n}$ converges in distribution to $S$. In the sequel, we will only be interested in the properties of the steady state discrete concentration $S$.

\subsection{Variability of the concentration}

One computes easily the mean $E_{d i s c}$ and variance $V_{d i s c}$ in the discretized model:

$$
\begin{aligned}
E_{\text {disc }} & :=\mathbb{E}(S)=\frac{D}{V_{d}} \frac{p}{1-a}, \\
V_{\text {disc }} & :=\mathbb{E}\left(S^{2}\right)-(\mathbb{E}(S))^{2}=\left(\frac{D}{V_{d}}\right)^{2} \frac{p-p^{2}}{1-a^{2}} .
\end{aligned}
$$

As we shall see in the next section, the discrete model tends in law to the continuous one when $p$ tends to 0 . For now, we simply remark that, indeed, $E_{\text {disc }}$ tends to $\mu \frac{D}{V_{d}}$ and $V_{\text {disc }}$ tends to $\frac{\mu}{2}\left(\frac{D}{V_{d}}\right)^{2}$ when $p$ tends to 0 , i.e. the mean and variance of (12) and (13) (note that, when $p \rightarrow 0, a \sim 1-\frac{p}{\mu}$ tends to 1).

For $E_{d i s c}$ constant, say $E_{\text {disc }}=1$, the variance in the discrete model equals $\frac{1-p}{p} \frac{1-a}{1+a}$. See Subsection 2.5 for a discussion of the limit cases $\lambda \rightarrow 0$ and $\lambda \rightarrow \infty$. As a consequence of Chebychev inequality we have (compare (16)):

$$
\mathbb{P}\left(\mid S-\mathbb{E}(S \mid \geqslant \gamma \mathbb{E}(S)) \leqslant \frac{1}{\gamma^{2}} \frac{1-p}{p} \frac{1-a}{1+a} .\right.
$$

In the sequel of this section, we will set $\frac{D}{V_{d}}=1$ for simplicity, as this quantity will not play any role in the rest of the analysis of Section 5. 
All the quantities above are functions of the discretization step $\frac{1}{n}$, or, equivalently, of $p$. We shall indicate this dependency by adding a subscript $p$ to the various quantities considered below, i.e. we write $S_{p}, \ldots$

$S_{p}$ is an infinite sum of independent Bernoulli random variables, thus its law is an infinite convolution of Bernoulli distribution, hence its name "infinite Bernoulli convolution"'. By writing:

$$
S=\frac{D}{V_{d}}\left(a X_{1}+\sum_{j=2}^{\infty} a^{j} X_{j}\right),
$$

and conditioning on the value of $X_{1}$, it is easily seen that the distribution $F_{p}$ of $S_{p}$ is the unique cumulative probability distribution solution to the self-similarity equation:

$$
F_{p}(x)=(1-p) F_{p}\left(\frac{x}{a}\right)+p F_{p}\left(\frac{x}{a}-1\right) .
$$

We denote by $m_{p}$ the associated measure, i.e. $m_{p}[0, x]=F_{p}(x)$. This measure has been studied in depth in fractal analysis as the invariant measure of the Iterated Function System (IFS) $\left(W_{1}, W_{2}\right)$ where $W_{1}(x)=a x, W_{2}(x)=a x+1$, and associated probability vector $(1-p, p)$.

By Fourier transforming (36), one gets that the characteristic function $\varphi_{p}$ of $S_{p}$ verifies:

$$
\varphi_{p}(\theta)=\left[1+p\left(e^{i a \theta}-1\right)\right] \varphi_{p}(a \theta),
$$

and, using the fact that $\varphi_{p}(0)=1$ :

$$
\varphi_{p}(\theta)=\prod_{j=1}^{\infty}\left[1+p\left(e^{i a^{j} \theta}-1\right)\right] .
$$

\subsection{Limit when the discretization step tends to 0}

In this subsection, we investigate the behaviour of $S_{p}$ when $n$ tends to infinity. Equivalently, we let $p$ (the probability of drug intake at any given time $\frac{i}{n}$ ) tend to 0 . Note that this also equivalent to letting $a$ tend to 1 .

We denote:

- $Y_{j}=a^{j} X_{j}$

- $S_{p}^{J}=\sum_{j=1}^{J} Y_{j}$. 
When $J$ tends to infinity, $S_{p}^{J}$ converge almost surely to $S_{p}=\sum_{j=1}^{\infty} Y_{j}$.

The following proposition shows that the discretized model tends to the continuous one defined in Section 3 when the discretization step tends to 0.

Proposition 7. $S_{p}=\sum_{j=1}^{\infty} Y_{j}$ tends in law to $C$ when $p$ tends to zero.

The proof of this result is given in section 8.3.

In practical terms, this means that, as long as we are only interested in distributional properties of the concentration, we may consider the discrete model instead of the continuous one provided $p$ is chosen small enough. Note however that the discrete model with arbitrary value of $p$ is interesting in its own right.

\subsection{Regularity of the limit distribution}

For each fixed $p, S_{p}$ is an infinite Bernoulli convolution. There is huge literature on the regularity of Bernoulli convolutions $[4,12,13,14,15,17]$. The regularity of the discretized model will display a sharp contrast depending on whether $a<\frac{1}{2}$ or $a \geqslant \frac{1}{2}$ :

- when $a<\frac{1}{2}$, the distribution of $S_{p}$ is singular with respect to the Lebesgue measure. More precisely, the Hausdorf dimension of its support is equal to $\frac{-\log (2)}{\log (a)}<1$ (recall that the Hausdorf dimension measures the "size", in a certain sense, of the support of the measure, i.e. where it is concentrated). This means that the set of possible values taken by the concentration is extremely sparse and does not form a continuum. In addition, the probability of being in an interval varies in a very non smooth way.

Note that $a<\frac{1}{2}$ is equivalent to $p>\mu \ln (2)$ or $h k>\ln (2)$ (recall that $h$ is the discretization step). This translates into the fact that, for fixed $k$, the time instants at which the patient is supposed to take his drugs are sufficiently spaced, and that he forgets to do so randomly at some of these instants. Alternatively, for a fixed $h$, the decay rate $k$ must be sufficiently large. Note also that, in view of (32), this is possible only if $\mu<\frac{1}{\log (2)}$. In this situation, the cumulative probability distribution of the concentration will thus be highly irregular : it will vary erratically, taking only very particular values, and the probability that it ranges in some interval varies wildly when the interval changes. 
- In contrast to the case $a<\frac{1}{2}$, where everything is known about the distribution of $S_{p}$, the case $a>\frac{1}{2}$ is much more delicate and is not completely settled, although great progress has been done in recent years. [17] (see also [14]) has proved that the distribution of $S_{p}$ is absolutely continuous for Lebesgue-almost all $a$ in $\left(\frac{1}{2}, 1\right)$ when $p=\frac{1}{2}$. This means that the distribution of the concentration has a probability density: in other words, the probability that the concentration ranges in some interval varies smoothly when the interval changes. This result has been extended to get absolute continuity for $p \in\left[\frac{1}{3}, \frac{2}{3}\right]$ and almost all $a \in\left[p^{p}(1-p)^{(1-p)}, 1\right)$ in [15]. On the other hand, the distribution is singular for all $a<p^{p}(1-p)^{(1-p)}$ without restrictions on $p$. In terms of pharmacokinetical parameters, singularity translates into:

$$
k h>-[\lambda h \log (\lambda h)+(1-\lambda h) \log (1-\lambda h)] .
$$

Unlike the case of the continuous model, regularity cannot be described solely in term of the ratio $\mu=\frac{\lambda}{k}$. Instead, both variables $\lambda$ and $k$ must be considered independently. However, in view of (38), it is natural to use the reduced quantities $\tilde{\lambda}:=\lambda h$ and $\tilde{k}=k h$. These are natural discrete counterparts of $\lambda$ and $k$ in this model. Figure 11 pictures the regions where the concentration behaves smoothly or erratically in the parameter space $(\tilde{\lambda}, \tilde{k})$.

The situation where the discretization step $h$ tends to 0 , or equivalently $a$ tends to 1 and $p$ tends to 0 is of particular interest to us, since, as shown above, the discretized model tends to the continuous one in this limit. Unfortunately, none of the results above about absolute continuity apply in our frame when we consider "small" $p$ : indeed, with $a=\exp (-p / \mu)$ and $p$ tending to zero, we have $a>p^{p}(1-p)^{(1-p)}$ for all sufficiently small $p$ irrespective of the value of $\mu$, as is easily shown by a Taylor expansion. Thus the condition ensuring singularity is not in force, while the result on absolute continuity result are not available because $p$ is too small. A recent result may however be of interest for us: [18] has proved absolute continuity for $p$ sufficiently small and almost all $a>1-0.6 p$. For small $p, a \sim 1-\frac{p}{\mu}$, and this hints that, for almost every value of $\mu$ above a certain threshold $\mu^{*}(p)$, such that $\mu^{*}(p) \rightarrow 5 / 3$ when $p \rightarrow 0$, the distribution of the concentration should have a density. Again, we find that a large $\mu$ translates into a better behaved concentration. 


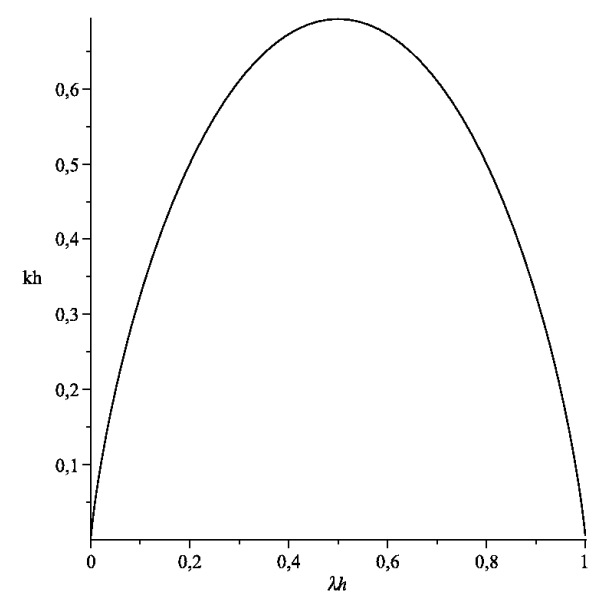

Figure 11: For values of $\tilde{k}=k h$ below the curve, the distribution of the concentration will be singular. Above the curve, and for $\tilde{\lambda}=\lambda h$ between $\frac{1}{3}$ and $\frac{2}{3}$, it will be absolutely continuous for almost all $k$.

\section{Discrete model with random instants and random doses}

In the discrete case, the random dose model takes the following form:

- We still have a sequence of i.i.d. Bernoulli random variables $\left(X_{j}\right)_{j \geqslant 1}$ with parameter $p$, which mark the random instants of drug intake.

- To account for the random dosing, we consider a sequence of i.i.d. random variables $\left(D_{j}\right)_{j \geqslant 1}$, such that again $D D_{j}$ will represent the doses. The $\left(D_{j}\right)_{j \geqslant 1}$ have distribution $\nu$ compactly supported on $\mathbf{R}_{+}^{*}$. We make the assumption that $\mathbb{E}\left(D_{j}\right)=1$, i.e., on average, the patient takes the prescribed dose. We also assume that the $D_{j}$ are independent of the $X_{j}$. We let $\left[d_{m}, d_{M}\right]$ denote the support of $\nu$. To avoid degenerate cases, we assume that, for any $\varepsilon>0$, there are an infinitely many indices $j, j^{\prime}$ for which $D_{j}<d_{m}+\varepsilon$ and $D_{j^{\prime}}>d_{M}-\varepsilon$.

- As previously, we set $a=e^{-p / \mu}$.

Using the same exchangeability argument as in section 5.1, we are led to consider the sum $\frac{D}{V_{d}} \sum_{j=1}^{n} a^{j} D_{j} X_{j}$, which converges almost surely (with respect to $\mathcal{B} \times \nu$, 
where $\mathcal{B}$ is the law of the $X_{j}$ ) to:

$$
S_{p}^{r d}=\frac{D}{V_{d}} \sum_{j=1}^{\infty} a^{j} D_{j} X_{j} .
$$

The characteristic function of $S_{p}^{r d}$ is easily obtained by conditioning on the values of $X_{j}$. Indeed,

$$
\mathbb{E}\left(e^{a^{j} D_{j} X_{j} \theta}\right)=1-p+p \varphi_{D}\left(a^{j} \theta\right)
$$

where $\varphi_{D}$ is the characteristic function of $D_{1}$. As a consequence:

$$
\varphi_{p}^{r d}(\theta)=\prod_{j=1}^{\infty}\left[1-p+p \varphi_{D}\left(a^{j} \theta\right)\right] .
$$

As before, we investigate the variability in this model, the limiting behaviour when $p$ tends to 0 , and finally the regularity of the distribution of the long-term concentration.

\subsection{Variability of the concentration}

One easily computes the mean and variance in this model:

\section{Proposition 8.}

$$
\begin{gathered}
E_{d i s c}^{r d}=E_{d i s c}=\frac{D}{V_{d}} \frac{p}{1-a}, \\
V_{d i s c}^{r d}=\left(\frac{\operatorname{Var}\left(D_{1}\right)}{1-p}+1\right) V_{d i s c} .
\end{gathered}
$$

This is similar to what was found in the continuous model (formula (26)), i.e. the added variability brought by random dosing enters in a multiplicative way. As noted in Subsection 2.6, the effect of random dosing on the variability is however amplified in the discrete model, due to the factor $\frac{1}{1-p}$.

Bounds like the ones in (14), (15) and (16) may then be obtained.

\subsection{Limit when the discretization step tends to 0}

Proposition 9. $S_{p}^{r d}$ converges in law to $C^{r d}$ when p tends to 0 .

The proof is similar to the one in the deterministic dose model, and is omitted. 


\subsection{Regularity of the limit distribution}

The problem of characterizing the regularity of the long term concentration is a hard one in general and we only give partial results of interest for us. Results recalled in Section 1 and Theorem 2 of [1], translated to our setting, entail the following:

Proposition 10. 1. If $a \geqslant \frac{d_{M}}{d_{m}+d_{M}}$, then the support of the concentration is an interval.

2. If $a<\frac{d_{m}}{d_{m}+d_{M}}$, then the support of the concentration is nowhere dense. Its Hausdorf dimension is equal to $-\frac{\log (2)}{\log (a)}$.

Note that, in the singular case, the dimension of the support is the same as in the deterministic dose case, i.e. random dosing does not increase irregularity.

The proof of this result is given in Section 8.4.

\section{A model with random elimination rate}

We consider a final model where not only the intake instants and doses are random, but also is the elimination rate: in other words, we take into account the fact that $k$ may take different values at different times. Of course, as noted in Subsection 2.7, the variations of $k$ will be very small for a typical patient. It is however interesting to investigate their impact on the variability and regularity of concentration, and to compare them with other effects of non compliance. As we will see, even small fluctuations of the elimination rate have a notable effect.

We will only consider a discrete version of the model. Precisely, our random time, random dose and random elimination model takes the following form:

- we still have a sequence of i.i.d. Bernoulli random variables $\left(X_{j}\right)_{j \geqslant 1}$ with parameter $p$, which mark the random instants of drug intake;

- random dosing is modeled by a sequence of i.i.d. random variables $\left(D_{j}\right)_{j \geqslant 1}$ as in section 6;

- to account for the random elimination rate, we replace the elimination factor $a=e^{-k h}$ by a sequence of i.i.d. random variables $\tilde{Z}_{i} \in(0,1)$ with mean $a$. In other words, at each discrete time, the elimination, instead of being fixed and equal to $a$, varies randomly "around" $a$. The condition on the support 
of the $\tilde{Z}_{i}$ is natural in view of the fact that they represent elimination rates. It will be more practical to consider the reduced random variables $Z_{i}=\frac{\tilde{Z}_{i}}{a}$. We write $G$ for the distribution of the $Z_{i}$. The constraints on $\tilde{Z}_{i}$ entail that $0<Z_{i}<\frac{1}{a}$ and $\mathbb{E}\left(Z_{i}\right)=1$. We note for further reference that this also implies:

$$
1 \leqslant \mathbb{E}\left(Z_{i}^{2}\right)<\frac{1}{a^{2}}
$$

We will let $\bar{G}$ denote the product measure $G^{\mathrm{N}}$.

We set $Y_{j}=\left(\prod_{n=1}^{j} Z_{n}\right) a^{j} D_{j} X_{j}$, and we investigate the behaviour of the almost sure limit (with respect to $\bar{G}$ ):

$$
S_{p}^{r e r d}=\frac{D}{V_{d}} \sum_{j=0}^{\infty} Y_{j}=\frac{D}{V_{d}} \sum_{j=0}^{\infty}\left(\prod_{n=0}^{j-1} Z_{n}\right) a^{j} D_{j} X_{j}
$$

Let us briefly explain how we obtain (42): the concentration at time $t_{n}=n h$ is equal to:

$$
C_{n}=\frac{D}{V_{d}} \sum_{j=0}^{n}\left(\prod_{i=j+1}^{n} \tilde{Z}_{i}\right) D_{j} X_{j}=\frac{D}{V_{d}} \sum_{j=0}^{n}\left(\prod_{i=j+1}^{n} a Z_{i}\right) D_{j} X_{j}
$$

By exchangeability, $C_{n}$ has the same law as $S_{n}$ :

$$
S_{n}=\frac{D}{V_{d}} \sum_{j=0}^{n}\left(\prod_{i=0}^{j-1} a Z_{i}\right) D_{j} X_{j}
$$

which indeed tends in law to $S_{p}^{\text {rerd }}$ given by (42), and is our random time, random dose and random elimination rate long term concentration. The distribution of this random variable coincides with the one of the invariant measure of the IFS with random maps $W_{1}(x)=Z a x, W_{2}(x)=Z a x+1$, and associated probability vector $(1-p, p)$, where $Z$ has distribution $G$. We shall denote by $F_{p}^{Z}$ the conditional distribution of $S_{p}^{r d}$ knowing $\left(Z_{1}, Z_{2}, \ldots\right)$. Such an IFS has been studied in depth in [13]. The assumptions ${ }^{4}$ in [13] (translated to our situation) are as follows:

1. All the $Z_{i}$ are independent of the $X_{j}$.

\footnotetext{
${ }^{4}$ the assumptions of [13] which are automatically satisfied in our setting are not mentioned here.
} 
2. The random variable $Z$ has an absolutely continuous distribution supported on $\mathbf{R}_{+}^{*}$, with density bounded from above by $\frac{C}{x}$ for all $x>0$, where $C$ is a constant.

3. The IFS is contracting on average, which translates into its Lyapunov $\xi$ exponent being negative. In our case, since the two contraction ratios are the same, this simply reads $\xi=\mathbb{E}(\log (Z))+\log (a)<0$.

Assumption (1) is certainly fulfilled in our frame: the random elimination rate does not depend on the fact that the patient takes his dose or not. The bounded density requirement in condition (2) is automatically verified here since $Z$ has compact support. Assumption (3) is also fulfilled, since $\mathbb{E}(Z)=1$ and, by Jensen inequality, this entails $\mathbb{E}(\log (Z)) \leqslant 0<p / \mu=-\log (a)$ (as mentioned above, requiring $\mathbb{E}(Z)=1$ is natural in our frame).

As before, we first proceed to investigate the variability in this model, and then the regularity of the distribution of the long-term concentration.

\subsection{Variability of the concentration}

\section{Proposition 11.}

$$
\begin{gathered}
E_{\text {disc }}^{r e r d}=E_{\text {disc }}=\frac{D}{V_{d}} \frac{p}{1-a} \\
V_{\text {disc }}^{\text {rerd }}=\left(\frac{D}{V_{d}}\right)^{2}\left[\frac{p}{1-a^{2} \mathbb{E}\left(Z^{2}\right)}\left(\mathbb{E}\left(D_{1}^{2}\right)+\frac{2 p a}{1-a}\right)-\left(\frac{p}{1-a}\right)^{2}\right] .
\end{gathered}
$$

The proof of this result is given in Section 8.5.

See Subsection 2.7 for an asymptotic analysis of the variance in the cases $\mu \rightarrow 0$ and $\mu \rightarrow \infty$ and a comparison between the various sources of randomness.

\subsection{Regularity of the limit distribution}

Theorem 2.1 and proposition 2.2 in [13] yield a complete answer regarding the regularity of $F_{p}^{Z}$ (i.e. the distribution conditioned on $\bar{G}$ ). Let $H=-p \log (p)-$ $(1-p) \log (1-p)$ denote the entropy of the measure $m_{p}$ associated to $F_{p}$. Then:

- if $-\xi<H, F_{p}^{Z}$ is absolutely continuous, $\bar{G}$-almost surely.

- if $-\xi \geqslant H, F_{p}^{Z}$ is singular, $\bar{G}$-almost surely. Its Haussdorf dimension is equal to $\frac{H}{-\xi}$. 
Translated to our setting, the condition for the distribution of the concentration to be (almost surely) singular is:

$$
\mathbb{E}(\log (Z))<p / \mu+p \log (p)+(1-p) \log (1-p),
$$

with the distribution being absolutely continuous when the reverse inequality holds.

Depending on the relative values of $\mathbb{E}(\log (Z)), \mu$, and $p$, one may thus observe two drastically distinct behaviours. Note however that, in the "interesting" cases $p \rightarrow 1$ and $p \rightarrow 0$, the distribution will always be singular, for a wide range of the admissible values of $\mathbb{E}(\log (Z))$ and $\mu$, or even irrespective of these values:

- When $p$ is large, we are in a situation where the instants at which the patient is supposed to take his drugs are sufficiently spaced in time. If $p$ is close to 1 , then $p / \mu+p \log (p)+(1-p) \log (1-p)$ is positive (for reasonable values of $\mu)$ and thus larger than $\mathbb{E}(\log (Z))$.

- When $p$ tends to 0 (i.e. in the continuous limit), $p / \mu+p \log (p)+(1-$ $p) \log (1-p)$ also tends to 0 , and thus, for any given distribution $G$, there exists $p_{0}=p_{0}(G)>0$ such that for all $p<p_{0}$, the distribution of concentration will be singular.

On the other hand, theorem 2.3 in [13] implies that, if:

$$
\mathbb{E}(\log (Z))>p / \mu-\mid \log \left(p^{2}+(1-p)^{2} \mid,\right.
$$

then the distribution will be $\bar{G}$-almost surely absolutely continuous with an $L^{2}$ density, and if:

$$
\mathbb{E}(\log (Z))>p / \mu-\frac{\mid \log \left(p^{2}+(1-p)^{2} \mid\right.}{2},
$$

then the distribution will be $\bar{G}$-almost surely absolutely continuous with a continuous density.

To sum up, we find that, in this model, the long term concentration will vary erratically in most cases of interest, and in particular for both $p$ large and small. Nonetheless, for moderate values of $p$ (for instance for $p=\frac{1}{2}$ ), and $\mu$ large enough (larger than 0.72 in the case $p=\frac{1}{2}$ ), one may find distributions of $Z$ ensuring that the concentration will be smooth.

Note finally that one could consider a (perhaps less natural) variant of the above model: instead of having a fixed law $G$ for the random dose perturbation, 
one may allow $G$ to depend on the time discretization. Denote $G_{p}$ this new family of laws. One could imagine that, in the continuous limit, the randomness on doses vanishes, i.e. $G_{p}$ tends to a Dirac at $Z=1$ when $p$ tends to 0 . In this case, and depending on the speed of convergence of $G_{p}$, one may use again Theorem 2.1 of [13] to prove that, conditionally on $\bar{G}_{p}$, the family of discrete distributions $F_{p}^{Z}$ will be, under some assumptions, absolutely continuous.

\section{Appendix}

\subsection{Proof of Proposition 5}

Fix $x>0$ and $\varepsilon>0$. Recall the inversion formula for characteristic functions:

$$
F(x+\varepsilon)-F(x)=\frac{1}{2 \pi} \lim _{T \rightarrow \infty} \int_{-T}^{T} \frac{e^{-i \theta(x+\varepsilon)}-e^{-i \theta x}}{-i \theta} \varphi(\theta) d \theta .
$$

Now,

$$
\begin{aligned}
\left|\int_{-1}^{1} \frac{e^{-i \theta(x+\varepsilon)}-e^{-i \theta x}}{-i \theta} \varphi(\theta) d \theta\right| & \leqslant \int_{-1}^{1} \frac{\left|e^{-i \theta \varepsilon}-1\right|}{\theta} d \theta \\
& \leqslant 2 \varepsilon
\end{aligned}
$$

and:

$$
\int_{1}^{T} \frac{e^{-i \theta(x+\varepsilon)}-e^{-i \theta x}}{-i \theta} \varphi(\theta) d \theta=\int_{1}^{T} \frac{e^{-i \theta(x+\varepsilon)}-e^{-i \theta x}}{-i \theta} \theta^{-\mu} d \theta+\int_{1}^{T} \frac{e^{-i \theta(x+\varepsilon)}-e^{-i \theta x}}{-i \theta} O\left(\theta^{-\mu-1}\right) d \theta
$$

But:

$$
\begin{aligned}
\left|\int_{1}^{T} \frac{e^{-i \theta(x+\varepsilon)}-e^{-i \theta x}}{-i \theta} O\left(\theta^{-\mu-1}\right) d \theta\right| & \leqslant K \int_{1}^{T} \frac{\left|e^{-i \theta \varepsilon}-1\right|}{\theta^{\mu+2}} d \theta \\
& \leqslant K \varepsilon \int_{1}^{T} \frac{1}{\theta^{\mu+1}} d \theta \\
& \leqslant K \varepsilon\left(1-\frac{1}{T^{\mu}}\right)
\end{aligned}
$$


so that

$$
\lim _{T \rightarrow \infty}\left|\int_{1}^{T} \frac{e^{-i \theta(x+\varepsilon)}-e^{-i \theta x}}{-i \theta} O\left(\theta^{-\mu-1}\right) d \theta\right| \leqslant K \varepsilon .
$$

Set now $\varepsilon=\varepsilon^{\prime} x$ then $u=\theta x$ to get:

$$
\begin{aligned}
\int_{1}^{T} \frac{e^{-i \theta(x+\varepsilon)}-e^{-i \theta x}}{-i \theta} \theta^{-\mu} d \theta & =\int_{1}^{T} \frac{e^{-i \theta x\left(1+\varepsilon^{\prime}\right)}-e^{-i \theta x}}{-i \theta^{\mu+1}} d \theta \\
& =i x^{\mu} \int_{x}^{x T} e^{-i u} \frac{e^{-i u \varepsilon^{\prime}}-1}{u^{\mu+1}} d u
\end{aligned}
$$

Integrating by parts, one gets

$$
\begin{aligned}
\int_{x}^{x T} e^{-i u} \frac{e^{-i u \varepsilon^{\prime}}-1}{u^{\mu+1}} d u= & {\left[i e^{-i u} \frac{e^{-i u \varepsilon^{\prime}}-1}{u^{\mu+1}}\right]_{x}^{x T} } \\
& -i \int_{x}^{x T} e^{-i u}\left(\frac{-i \varepsilon^{\prime} e^{-i u \varepsilon^{\prime}}}{u^{\mu+1}}-\frac{(\mu+1)\left(e^{-i u \varepsilon^{\prime}}-1\right)}{u^{\mu+2}}\right) d u .
\end{aligned}
$$

We have:

$$
\left[i e^{-i u} \frac{e^{-i u \varepsilon^{\prime}}-1}{u^{\mu+1}}\right]_{x}^{\infty}=i e^{-i x} \frac{e^{-i x \varepsilon^{\prime}}-1}{x^{\mu+1}}=O(\varepsilon)
$$

when $\varepsilon \rightarrow 0$.

Since the integral $\int_{x}^{\infty} e^{-i u} \frac{e^{-i u \varepsilon^{\prime}}}{u^{\mu+1}} d u$ is convergent, the term $\int_{x}^{\infty} e^{-i u-i \varepsilon^{\prime} e^{-i u \varepsilon^{\prime}}} \frac{u^{\mu+1}}{d u}$ is also $O(\varepsilon)$. Finally, the term $\int_{x}^{\infty} e^{-i u} \frac{(\mu+1)\left(e^{-i u \varepsilon^{\prime}}-1\right)}{u^{\mu+2}} d u$ is dealt with as above to show that it is again $O(\varepsilon)$.

\subsection{Proof of Proposition 6}

Thanks to our assumption on $\nu,(23)$ yields:

$$
\begin{aligned}
\varphi_{t}^{r d}(\theta) & =\exp \left\{\lambda \int_{0}^{t} \int_{A}\left[\exp \left(i \theta \frac{D}{V_{d}} u e^{-k(t-x)}\right)-1\right] \nu(d u) d x\right\} \\
& =\exp \left\{\lambda \int_{A} \int_{0}^{t}\left[\exp \left(i \theta \frac{D}{V_{d}} u e^{-k(t-x)}\right)-1\right] d x \nu(d u)\right\}
\end{aligned}
$$


Setting $y=\theta \frac{D}{V_{d}} u e^{-k(t-x)}$, we get:

$$
\varphi_{t}^{r d}(\theta)=\exp \left\{\int_{A} \mu \int_{\theta \frac{D}{V_{d}} u e^{-k t}}^{\theta \frac{D}{V_{d}} u} \frac{e^{i y}-1}{y} d y \nu(d u)\right\} .
$$

Letting $t \rightarrow \infty$,

$$
\varphi^{r d}(\theta)=\exp \left\{\int_{A} \mu \int_{0}^{\theta \frac{D}{V_{d}} u} \frac{e^{i y}-1}{y} d y \nu(d u)\right\} .
$$

From the proof of proposition 4, we know that, when $\theta$ tends to infinity,

$$
\mu \int_{0}^{\theta \frac{D}{V_{d}} u} \frac{e^{i y}-1}{y} d y \sim K-\mu(\log (\theta)+\log (u))
$$

for a certain constant $K$, with in addition $\mu \int_{0}^{\theta \frac{D}{V_{d}} u} \frac{e^{i y}-1}{y} d y-K+\mu(\log (\theta)+\log (u))$ tending to 0 . Thus, using the assumption on the logarithmic moment of $\nu$,

$$
\int_{A} \mu \int_{0}^{\theta \frac{D}{V_{d}} u} \frac{e^{i y}-1}{y} d y \nu(d u) \sim K-\mu \log (\theta)-\mu \int_{A} \log (u) \nu(d u)
$$

and one finishes up the proof with the help of a dominated convergence argument to show that the difference between the right-hand side and the left-hand side in the equivalent above indeed tends to 0 when $\theta$ tends to infinity.

\subsection{Proof of Proposition 7}

We shall prove Proposition 7 by showing that the characteristic function $\varphi_{p}$ of $S_{p}$ tends pointwise to the characteristic function $\varphi$ of $C$.

We first collect two technical lemmas that will be needed in the sequel.

Lemma 12. Let $z \in \mathbb{C}$ be such that $|z| \leqslant 1 / 2$. Then $\log (1+z)=z+r(z)$ with $|r(z)| \leqslant|z|^{2}$, where Log denotes the principal value of the logarithm.

Proof.

We integrate $\frac{d \zeta}{\zeta}$ over the segment $[1,1+z]$, parametrized by $\zeta=1+z t$ :

$$
\log (1+z)=\int_{1}^{1+z} \frac{d \zeta}{\zeta}=\int_{0}^{1} \frac{z}{1+t z} d t
$$


Thus:

$$
\begin{gathered}
r(z)=\log (1+z)-z=\int_{0}^{1} \frac{z}{1+t z} d t-\int_{0}^{1} z d t=-\int_{0}^{1} \frac{t z^{2}}{1+t z} \\
\text { If }|z| \leqslant 1 / 2 \text {, then }|1+t z| \geqslant 1 / 2 \text { and } \\
\qquad|r(z)| \leqslant \int_{0}^{1}\left|\frac{t z^{2}}{1+t z}\right| d t \leqslant 2|z|^{2} \int_{0}^{1} t d t=|z|^{2}
\end{gathered}
$$

For any function $f$ defined on $\mathbb{R}_{+}$, and for all natural numbers $n, k \geqslant 1$, let $S(f, n, k)$ denote the Riemann sum of $f$ over $[0, k]$ with step $1 / n$ :

$$
S(f, n, k)=\frac{1}{n} \sum_{j=1}^{n k} f\left(\frac{j}{n}\right)
$$

Provided $f$ is sufficiently regular and integrable, one has $\lim _{n \rightarrow \infty} S(f, n, k)=$ $\int_{0}^{k} f(t) d t$. By dominated convergence, $\lim _{k \rightarrow \infty} \lim _{n \rightarrow \infty} S(f, n, k)=\int_{0}^{\infty} f(t) d t$. The next lemma gives a sufficient condition on an increasing sequence of integers $\left(k_{n}\right)_{n \in \mathbf{N}}$ ensuring that $\lim _{n \rightarrow \infty} S\left(f, n, k_{n}\right)=\int_{0}^{\infty} f(t) d t$.

Lemma 13. Let $f$ be defined on $\mathbb{R}_{+}$. Assume $f$ is Lipschitzian and integrable. If $k_{n}=o(n), n \rightarrow \infty$, then $S\left(f, n, k_{n}\right)$ converges to the integral of $f$ when $n$ tends to infinity.

Proof.

Write:

$S\left(f, n, k_{n}\right)=\int_{0}^{\infty} \sum_{j=1}^{n k_{n}} f\left(\frac{j}{n}\right) \mathbb{1}_{[(j-1) / n, j / n[}(t) d t$.

One has:

$$
S\left(f, n, k_{n}\right)-\int_{0}^{\infty} f(t) d t=\sum_{j=1}^{n k_{n}} \int_{(j-1) / n}^{j / n}\left(f\left(\frac{j}{n}\right)-f(t)\right) d t-\int_{k_{n}}^{\infty} f(t) d t
$$


The last term in the right hand side above tends to 0 by dominated convergence. The absolute value of each of the $n k_{n}$ terms in the sum is bounded by $\frac{1}{n} \omega\left(\frac{1}{n}\right)$, where $\omega$ is the modulus of continuity of $f$. This implies that the absolute value of the sum is not larger than $k_{n} \omega\left(\frac{1}{n}\right) \leqslant C k_{n} / n$, which tends to 0 by assumption when $n$ tends to infinity.

Remark. The proof above shows that the conclusion of the lemma holds for $k_{n}=$ $o(1 / \omega(1 / n))$.

Proof of proposition 7.

Recall that $\varphi_{p}$ denotes the characteristic function of $S_{p}$, and let $\varphi_{Y_{j}}$ denote the one of $Y_{j}$. One has:

$$
\varphi_{p}(\theta)=\prod_{j=1}^{\infty} \varphi_{Y_{j}}(\theta)=\prod_{j=1}^{\infty}\left(1+p\left(e^{i a^{j} \theta}-1\right)\right) .
$$

For $p$ small enough, all the $1+p\left(e^{i a^{j} \theta}-1\right)$ lie in the half-plane $\mathfrak{R e} z>0$, and we may permute the logarithm and the product. In addition, again for $p$ small enough, $\left|p\left(e^{i a^{j} \theta}-1\right)\right|<\frac{1}{2}$ so that we may apply Lemma 12 to get $\log \varphi_{p}(\theta)=$ $\sum_{j=1}^{\infty} \log \left(1+p\left(e^{i a^{j} \theta}-1\right)\right)=\sum_{j=1}^{\infty}\left[p\left(e^{i a^{j} \theta}-1\right)+r\left(p\left(e^{i a^{j} \theta}-1\right)\right)\right]$.

Denote $\psi_{p}(\theta)=p \sum_{j=1}^{\infty} e^{i a^{j} \theta}-1$ and $R_{p}(\theta)=\sum_{j=1}^{\infty} r\left(p\left(e^{i a^{j} \theta}-1\right)\right)$.

We first obtain an upper bound for the remainder term $R_{p}$ using the fact that $|r(z)| \leqslant|z|^{2}:\left|R_{p}(\theta)\right| \leqslant \sum_{j=1}^{\infty} p^{2}\left|e^{i a^{j} \theta}-1\right|^{2}$. Since $e^{i a^{j} \theta}-1=2 i e^{i a^{j} \theta / 2} \sin \left(a^{j} \theta / 2\right)$, one has $\left|e^{i a^{j} \theta}-1\right|^{2}=4 \sin ^{2}\left(a^{j} \theta / 2\right) \leqslant a^{2 j} \theta^{2}$ and

$$
\left|R_{p}(\theta)\right| \leqslant p^{2} \theta^{2} \sum_{j=1}^{\infty} a^{2 j}=\frac{p^{2} \theta^{2} a^{2}}{1-a^{2}}
$$


Apply the inequality $1-e^{-u} \geqslant u e^{-u}$, valid for all $u \geqslant 0$, with $u=2 p / \mu$ to get $1-a^{2}=1-e^{-2 p / \mu} \geqslant 2 \frac{p}{\mu} e^{-2 p / \mu}=2 \frac{p}{\mu} a^{2}$. Finally,

$$
\left|R_{p}(\theta)\right| \leqslant \mu \frac{p \theta^{2}}{2},
$$

which shows that $\lim _{p \rightarrow 0} R_{p}(\theta)=0$ uniformly on all compact sets.

Let us now deal with $\psi_{p}$. Write $p=p_{n}=c / n$ and thus $a=a_{n}=e^{-c / \mu n}$, where $c \in(0,1 / 2)$ is a constant. Denote $k_{n}=\lfloor\sqrt{n}\rfloor, A=A_{n}=n k_{n}$ and define: $\psi_{p}(\theta)=: \psi_{p}^{1}(\theta)+\psi_{p}^{2}(\theta)$ where

$$
\begin{gathered}
\psi_{p}^{1}(\theta)=p \sum_{j=1}^{A} e^{i a^{j} \theta}-1, \\
\psi_{p}^{2}(\theta)=p \sum_{j=A+1}^{\infty} e^{i a^{j} \theta}-1 .
\end{gathered}
$$

Consider first $\psi_{p}^{2}$ :

$$
\left|\psi_{p}^{2}(\theta)\right| \leqslant p \sum_{j=A+1}^{\infty}|\theta| 2 a^{j}=\frac{2 p|\theta| a^{A+1}}{1-a} \leqslant \frac{2 p \mu|\theta| a^{A+1}}{p a}=2 \mu|\theta| a^{A} .
$$

Since $a=e^{-c / \mu n}$ and $A=n \sqrt{n}\left(1+\varepsilon_{n}\right)$ :

$$
a^{A}=e^{-c \sqrt{n}\left(1+\varepsilon_{n}\right) / \mu} \leqslant e^{-2 c \sqrt{n} / \mu},
$$

as soon as $n$ is sufficiently large to ensure that $\left|\varepsilon_{n}\right| \leqslant 1$. As a consequence, $\lim _{n \rightarrow \infty} \psi_{2}^{p}(\theta)=0$ uniformly on all compact sets.

We will estimate the limit of $\psi_{p}^{1}$ with the help of Lemma 13. Write:

$$
\psi_{p}^{1}(\theta)=p \sum_{j=1}^{A} e^{i a^{j} \theta}-1=\frac{c}{n} \sum_{j=1}^{n k_{n}} e^{i \theta e^{-c j / \mu n}}-1 .
$$


This is equal to $c S\left(f, n, k_{n}\right)$ with $f(t)=e^{i \theta e^{-c t / \mu}}-1$. This function is continuous on $\mathbb{R}_{+}$. Furthermore, since:

$$
|f(t)| \leqslant 2 \sin e^{-c t / \mu}|\theta| \leqslant 2 e^{-c t / \mu}|\theta|,
$$

and $c / \mu>0, f$ is integrable. Finally, it is differentiable and one computes:

$$
f^{\prime}(t)=-i \frac{c}{\mu} \theta e^{-c t / \mu} e^{i \theta e^{-c t / \mu}}
$$

thus

$$
\left|f^{\prime}(t)\right|=\frac{c}{\mu}|\theta| e^{-c t / \mu} \leqslant \frac{c}{\mu}|\theta|<\infty .
$$

This shows that $f$ is a Lipschitz function. Noting that $k_{n}=o(n)$, we may apply Lemma 13 to obtain that $S\left(f, n, k_{n}\right)$ converges to the integral of $f$.

Gathering our results, we get:

$$
\begin{aligned}
\lim _{n \rightarrow \infty} \varphi_{p_{n}}(\theta) & =\exp \left(c \int_{0}^{\infty}\left(e^{i \theta e^{-c t / \mu}}-1\right) d t\right) \\
& =\exp \left(\int_{0}^{\infty}\left(e^{i \theta e^{-v / \mu}}-1\right) d v\right) \\
& =\exp \left(\mu \int_{0}^{\theta} \frac{e^{i u}-1}{u} d u\right) .
\end{aligned}
$$

\subsection{Proof of Proposition 10}

Consider a generalized Bernoulli convolution of the form $S=\sum_{k=1}^{\infty} b_{k} X_{k}$, where $\left(X_{k}\right)$ are Bernoulli random variables as above and $\left(b_{k}\right)$ is a sequence of positive real numbers such that $\sum_{k=1}^{\infty} b_{k}<\infty$. Then [1]:

- If, for all $k, b_{k} \leqslant \sum_{j=k+1}^{\infty} b_{j}$, the support of $S$ is a closed interval.

- If for all large enough $k, b_{k}>\sum_{j=k+1}^{\infty} b_{j}$, the support of $S$ is a nowhere dense set. 
- If for all large enough $k, b_{k} \geqslant \sum_{j=k+1}^{\infty} b_{j}$, then $S$ has a singular density and the Hausdorf dimension of its support is $\underline{\lim }_{n \rightarrow \infty} n \frac{-\log (2)}{\log \left(\sum_{j=n+1}^{\infty} b_{j}\right)}$.

We apply these results with $b_{k}=a^{k} D_{k}$. Then, for all realizations of the $\left(D_{j}\right)_{j}$, we have that $b_{k} \leqslant a^{k} d_{M}$ and $\sum_{j=k+1}^{\infty} b_{j} \geqslant d_{m} \sum_{j=k+1}^{\infty} a^{j}=d_{m} \frac{a^{k+1}}{1-a}$. A sufficient condition for $b_{k} \leqslant \sum_{j=k+1}^{\infty} b_{j}$ is thus that $a \geqslant \frac{d_{M}}{d_{m}+d_{M}}$. The other case follows in a similar way. Note that:

$$
d_{m} \frac{a^{n+1}}{1-a} \leqslant \sum_{j=n+1}^{\infty} b_{j} \leqslant d_{M} \frac{a^{n+1}}{1-a},
$$

which entails the formula above for the Hausdorf dimension.

\subsection{Proof of Proposition 11}

Verifying the expression of the mean is straightforward (recall that $\mathbb{E}(Z)=1$ ).

For the variance, note that:

$\left(S_{p}^{r e r d}\right)^{2}=\left(\frac{D}{V_{d}}\right)^{2}\left[\sum_{j=0}^{\infty}\left(\prod_{n=0}^{j-1} Z_{n}^{2}\right) a^{2 j} D_{j}^{2} X_{j}^{2}+2 \sum_{j<k}\left(\prod_{n=0}^{j-1} Z_{n}\right)\left(\prod_{n=0}^{k-1} Z_{n}\right) a^{j+k} D_{j} D_{k} X_{j} X_{k}\right]$.

Now, for $j<k$,

$$
\begin{gathered}
\mathbb{E}\left[\left(\prod_{n=0}^{j-1} Z_{n}^{2}\right)\right]=\mathbb{E}\left(Z^{2}\right)^{j}, \\
\mathbb{E}\left[\left(\prod_{n=0}^{j-1} Z_{n}\right)\left(\prod_{n=0}^{k-1} Z_{n}\right)\right]=\mathbb{E}\left[\left(\prod_{n=0}^{j-1} Z_{n}^{2}\right)\left(\prod_{n=j}^{k-1} Z_{n}\right)\right]=\mathbb{E}\left(Z^{2}\right)^{j},
\end{gathered}
$$

and thus 


$$
\begin{aligned}
\mathbb{E}\left[\left(S_{p}^{\text {rerd }}\right)^{2}\right] & =\left(\frac{D}{V_{d}}\right)^{2}\left[\sum_{j=0}^{\infty} \mathbb{E}\left(Z^{2}\right)^{j} a^{2 j} \mathbb{E}\left(D_{1}^{2}\right) p+2 \sum_{j<k} \mathbb{E}\left(Z^{2}\right)^{j} a^{j+k} p^{2}\right] \\
& =\left(\frac{D}{V_{d}}\right)^{2} p\left[\sum_{j=0}^{\infty} \mathbb{E}\left(Z^{2}\right)^{j} a^{j}\left(a^{j} \mathbb{E}\left(D_{1}^{2}\right)+2 p \sum_{k=j+1}^{\infty} a^{k}\right)\right] \\
& =\left(\frac{D}{V_{d}}\right)^{2} p\left[\sum_{j=0}^{\infty} \mathbb{E}\left(Z^{2}\right)^{j} a^{j}\left(a^{j} \mathbb{E}\left(D_{1}^{2}\right)+2 p \frac{a^{j+1}}{1-a}\right)\right] \\
& =\left(\frac{D}{V_{d}}\right)^{2} p\left[\mathbb{E}\left(D_{1}^{2}\right) \sum_{j=0}^{\infty} \mathbb{E}\left(Z^{2}\right)^{j} a^{2 j}+2 \frac{p a}{1-a} \sum_{j=0}^{\infty} \mathbb{E}\left(Z^{2}\right)^{j} a^{2 j}\right] \\
& =\left(\frac{D}{V_{d}}\right)^{2} \frac{p}{1-a^{2} \mathbb{E}\left(Z^{2}\right)}\left[\mathbb{E}\left(D_{1}^{2}\right)+2 \frac{p a}{1-a}\right] .
\end{aligned}
$$

Note that the series $\sum_{j=0}^{\infty} \mathbb{E}\left(Z^{2}\right)^{j} a^{2 j}$ does indeed converge, since $Z$ is supported on $(0,1 / a)$.

\section{References}

[1] Albeverio, S., Torbin, G. (2008). On fine fractal properties of generalized infinite Bernoulli convolutions. Bull.Sci. Math. 132, 711-727.

[2] Barrière, O., Li, J. and Nekka, F. (2011). A Bayesian approach for the estimation of patient compliance based on the last sampling information. J. Pharmacokinet. Pharmacodyn. 38 (3), 333-351.

[3] Bingham, N. H., Goldie, C.M. and Teugels, J.L. (1989). Regular Variation, Cambridge University Press.

[4] Erdós, P. (1939). On a family of symmetric Bernoulli convolutions, Amer. J. Math. 61, 974-975.

[5] Feller, W. (1971). An introduction to probability theory and its applications, Vol. II, 3rd edition, Wiley. 
[6] Greenblatt, DJ, Harmatz, JS, Friedman, H, Locniskar, A and Shader, RI. (1989). A large-sample study of diazepam pharmacokinetics, The Drug Monit. 11 (6), 652-657.

[7] Iskedjian, M., Einarson, T.R., MacKeigan, L.D., Shear, N., Addis, A., Mittmann, N., and Ilersich, A.L. (2002). Relationship between daily dose frequency and adherence to antihypertensive pharmacotherapy: evidence from a meta-analysis. Clin. Ther. 24 (2), 302-316.

[8] Kingman, J.F.C. (1993). Poisson Processes, Oxford University Press, Oxford.

[9] Li, J. and Nekka, F. (2007). A Pharmacokinetic Formalism Explicitly Integrating the Patient Drug Compliance. J. Pharmacokinet. Pharmacodyn. 34 (1), 115-139.

[10] Li, J. and Nekka, F. (2009). A Probabilistic Approach for the Evaluation of Pharmacological Effect Induced by Patient Irregular Drug Intake. J. Pharmacokinet. Pharmacodyn. 36 (3), 221-246.

[11] Loeve, M., Probability Theory I, Fourth Edition, Springer Verlag, 1977.

[12] Peres, Y., Schlag, W., Solomyak, B. (2000). Sixty years of Bernoulli convolutions, Fractal geometry and stochastics, II (Greifswald/Koserow, 1998), Progr. Probab., Vol. 46, Birkhäuser, Basel, 39-65.

[13] Peres, Y. and Simon, K. and Solomyak, B. (2006). Absolute Continuity for random iterated function systems with overlaps, J. London Math. Soc. (2) 74, 739-756.

[14] Peres, Y. and Solomyak, B. (1996). Absolute Continuity of Bernoulli Convolution, a Simple Proof, Math. Res. Lett. 3 (2), 231-239.

[15] Peres, Y. and Solomyak, B. (1998). Self-Similar Measures and Intersections of Cantor Sets, Trans. Amer. Math. Soc. 350 (10), 4065-4087.

[16] Petrov, V., Limit Theorems of Probability Theory, Oxford Science Publication, 1995.

[17] Solomyak, B. (1995). On the random series $\sum \pm \lambda^{i}$ (an Erdós problem), Annals of Math. 142, 611-625. 
[18] Toth, H.R. (2008). Infinite Bernoulli convolutions with different probabilities, Discrete and Continuous Dynamical Systems 21 (2), 595-600.

[19] Tsybakov, A. (2010). Introduction to Nonparametric Estimation, Springer Series in Statictics.

[20] World Health Organization (2003). Adherence to Long-term therapies: Evidence for action. http://www.who.int/chronic conditions/adherencereport/en/. 